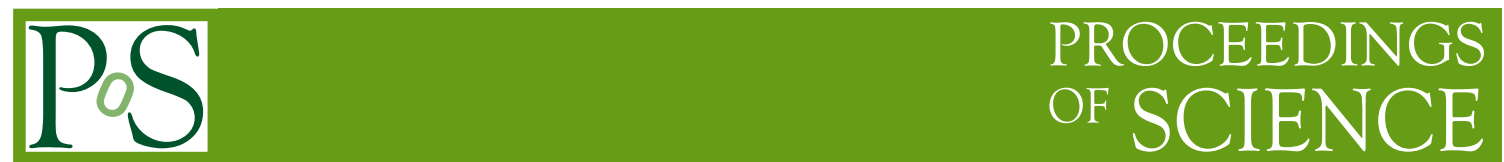

\title{
Black-Hole Phenomenology
}

\author{
Neven Bilić* \\ Rudjer Bošković Intitute, Zagreb, Craotia \\ E-mail: bilicethphys.irb.hr
}

This set of lectures is an introduction to black-hole astrophysics. The emphasis is made on the phenomenology of X-ray binaries and of supermassive compact objects at galactic centers.

School on Particle Physics, Gravity and Cosmology

August 21 -September 2, 2006

Dubrovnik

*Speaker. 


\section{Contents}

$\begin{array}{lll}\text { 1. Introduction } & 4\end{array}$

2. Preliminaries 4

2.1 Spherical Configurations 4

2.2 Schwarzschild Solution $\quad 5$

2.2.1 Birkhoff's Theorem 6

$\begin{array}{lll}2.3 & \text { Spherical Stars } & 6\end{array}$

2.4 Newtonian Limit 6

$\begin{array}{lll}2.4 .1 & \text { Polytropes } & 7\end{array}$

3. Compact Astrophysical Objects $\quad 8$

3.1 White Dwarfs 8

3.1.1 Equation of State $\quad 8$

$\begin{array}{llr}3.2 & \text { Neutron Stars } & 10\end{array}$

$\begin{array}{ll}3.3 \text { Black Holes } & 12\end{array}$

$\begin{array}{lll}\text { 3.3.1 Spherical Collapse } & 13\end{array}$

$\begin{array}{ll}\text { 3.3.2 Eddington-Finkelstein Coordinates } & 15\end{array}$

4. Rotating Black Holes 16

$\begin{array}{lll}4.1 & \text { Geodesic Motion } & 17\end{array}$

$\begin{array}{lll}\text { 4.1.1 Photon Circular Orbit } & 18\end{array}$

$\begin{array}{ll}\text { 4.1.2 Angular Velocity } & 18\end{array}$

4.1.3 Innermost Stable Circular Orbit $\quad 18$

$\begin{array}{lll}4.2 & \text { Ergosphere } & 19\end{array}$

$\begin{array}{ll}\text { 4.2.1 Penrose Process } & 20\end{array}$

5. Stellar Mass BHs versus NSs $\quad 21$

$\begin{array}{lll}5.1 \text { X-ray Binaries } & 21\end{array}$

$\begin{array}{lll}\text { 5.1.1 Binary X-ray Pulsars } & 21\end{array}$

5.1.2 Mass Measurements $\quad 22$

$\begin{array}{lll}5.2 & \text { Black-Hole Binaries } & 23\end{array}$

5.2.1 Cygnus X-1: A Black-Hole Candidate 23

$\begin{array}{lll}5.3 & \text { Spin Estimates } & 24\end{array}$

6. Supermassive Black Holes 26

6.1 Masses and Radii 26

$\begin{array}{lll}\text { 6.1.1 Gas Spectroscopy } & 27\end{array}$

$\begin{array}{lll}\text { 6.1.2 Maser Interferometry } & 27\end{array}$

$\begin{array}{lll}\text { 6.1.3 Virial Mass } & 27\end{array}$

$\begin{array}{ll}6.2 \text { Sagittarius } \mathrm{A}^{*} & 28\end{array}$ 
6.3 Supermassive BHs in Active Galactic Nuclei 29

6.3.1 Eddington Limit 29

$\begin{array}{ll}\text { 6.3.2 Radius and Mass Estimate } & 31\end{array}$

6.3.3 Estimate of the AGN Efficiency 32

7. Intermediate Mass BHs 32

8. Observational Evidence for the Horizon 32

8.1 ADAF 32

8.2 X-ray Bursts 33

8.3 Direct Imaging 33

9. Alternatives to Supermassive BHs

9.1 Neutrino Stars 34

9.2 Boson Stars 35

9.3 Dark-Energy Stars 38

$\begin{array}{lll}\text { 9.3.1 De Sitter Gravastars } & 38\end{array}$

9.3.2 Chaplygin Gravastars 39

A. Basic General Relativity 41

A.1 Geometry 41

A.2 Covariant Derivative 42

A.3 Geodesics 42

A.4 Isometries and Killing Vectors 43

A.5 Constants of Motion 43

A.6 Einstein's Equations 44

B. Basic Fluid Dynamics $\quad 44$

B.1 Fluid Velocity 45

B.2 Hydrostatic Equilibrium 45

C. Basic Thermodynamics 46

C.1 Tolman Equations 46

$\begin{array}{lll}\text { C.2 } & \text { Fermi Distribution } & 47\end{array}$

$\begin{array}{lll}\text { C.3 Isentropic Fluid } & 48\end{array}$

C.4 Degenerate Fermi Gas 48

C.5 Polytropic Gases 49 


\section{Introduction}

Black holes $(\mathrm{BH})$ are the most fascinating objects predicted by general relativity. There exist about 20 confirmed candidates [1] for astrophysical BHs in the mass range $5-20 M_{\odot}$ and about three dozen supermassive BH candidates [2] in the mass range $10^{6}-10^{9.5} M_{\odot}$.

Unfortunately, there exists as yet no direct evidence for astrophysical BHs ${ }^{1}$. At present we only hope that the black-hole paradigm may be proved or ruled out by comparing $\mathrm{BH}$ candidates with credible alternatives. Fortunately, BHs are dark and compact, which narrows the list of possible alternatives among standard astrophysical objects. For a stellar-mass $\mathrm{BH}$, the only standard astrophysical alternative is neutron stars (NS). It is therefore quite important to understand the properties of NSs and their observational distinction to BHs. Therefore, considerable attention will be devoted here to NSs.

These lecture notes are in large part based on the standard text books Misner, Thorne, and Wheeler [3], Shapiro and Teukolsky [4], Wald [5], and Carroll and Ostlie [6], and on the review articles by Townsend [7] and Narayan [8]. The exception is section 9 which is based on original articles. Efforts are made to provide citations to original papers wherever appropriate but the list of references is by no means complete. A number of interesting talks may be found at the site of a recent conference on supermassive black holes [9].

We use the positive-time negative-space signature convention, i.e., (+,- - -) and we mostly use the so-called natural units in which $c=\hbar=G=1$. In these units, the physical quantities are expressed in powers of the Planck mass $m_{\mathrm{Pl}}=\sqrt{\hbar c / G}$, Planck length $l_{\mathrm{Pl}}=\sqrt{\hbar G / c^{3}}$, or Planck time $t_{\mathrm{Pl}}=l_{\mathrm{Pl}} / c$.

\section{Preliminaries}

\subsection{Spherical Configurations}

Consider the space time metric

$$
d s^{2}=g_{\mu v} d x^{\mu} d x^{v}
$$

in which the metric tensor $g_{\mu \nu}$ is time independent. In general relativity we distingush between static and stationary metric. Stationary configurations are described by the metric coefficients that do not depend on time. For static configurations we also require the time reversal $(t \rightarrow-t)$ invariance of the metric. In this case, the mixed components $g_{0 i}$ must vanish.

Static fluid configurations are spherical. The most general metric generated by a spherical mass distribution is of the form

$$
d s^{2}=\xi^{2} d t^{2}-\lambda^{2} d r^{2}-r^{2}\left(d \theta^{2}+\sin ^{2} \theta d \phi^{2}\right),
$$

where $\xi$ and $\lambda$ are functions of $r$ only. The function $\xi$, called the "lapse function", may be represented in terms of the gravitational potential

$$
\xi=e^{\varphi(r)}
$$

\footnotetext{
${ }^{1}$ See A. Müller's lecture at this School
} 
and the function $\lambda$ is related to the enclosed mass $\mathscr{M}(r)$

$$
\lambda=\left(1-\frac{2 \mathscr{M}(r)}{r}\right)^{-1 / 2} .
$$

It may be easily shown [10] that Einstein's equations reduce to two nontrivial independent equations

For a perfect fluid

$$
\begin{gathered}
\frac{2}{\xi} \frac{d \xi}{d r}=-8 \pi r \lambda^{2} T_{r}^{r}+\frac{\lambda^{2}-1}{r} \\
\frac{2}{\lambda} \frac{d \lambda}{d r}=8 \pi r \lambda^{2} T_{0}^{0}-\frac{\lambda^{2}-1}{r} .
\end{gathered}
$$

$$
T^{r}{ }_{r}=-p, \quad T_{0}^{0}=\rho .
$$

Using (2.4), Einstein's field equations take the form

$$
\begin{gathered}
\frac{d \xi}{d r}=\xi \frac{\mathscr{M}+4 \pi r^{3} p}{r(r-2 \mathscr{M})} \\
\frac{d \mathscr{M}}{d r}=4 \pi r^{2} \rho .
\end{gathered}
$$

The latter may be written in the form

$$
\mathscr{M}(r)=\int_{0}^{r} d r^{\prime} 4 \pi r^{2} \rho\left(r^{\prime}\right),
$$

which shows that the function $\mathscr{M}$ may indeed be interpreted as an enclosed mass.

Finally, if we impose the particle number conservation constraint (C.1), we have

$$
\int_{0}^{R} d r 4 \pi r^{2}(1-2 \mathscr{M} / r)^{-1 / 2} n(r)=N
$$

where we have employed the spherical symmetry to replace the proper volume integral as

$$
\int_{\Sigma} u^{\mu} d \Sigma_{\mu}=\int_{0}^{R} d r 4 \pi r^{2} \lambda
$$

\subsection{Schwarzschild Solution}

Assume the absence of matter in the region $r>R$. In this region equations (2.8) and (2.9) may be easily solved. One finds

$$
\begin{gathered}
\mathscr{M}(r)=M=\text { const. } \\
\xi(r)=\left(1-\frac{2 M}{r}\right)^{1 / 2}
\end{gathered}
$$

The metric takes the form

$$
d s^{2}=\left(1-\frac{2 M}{r}\right) d t^{2}-\left(1-\frac{2 M}{r}\right)^{-1} d r^{2}-r^{2}\left(d \theta^{2}+\sin ^{2} \theta d \phi^{2}\right) .
$$

This is known as the Schwarzschild metric. The constant $M$ is the mass of the source. In the weak field limit $r \gg M$, we obtain the Newtonian potential

$$
\varphi=\ln \xi \approx-\frac{M}{r} .
$$

This metric describes the gravitational field outside of any spherical object of mass $M$, including a black hole. The sphere with radius $r=2 M$, at which the metric diverges, is the $\mathrm{BH}$ event horizon. 


\subsubsection{Birkhoff's Theorem}

Theorem 1. The exterior spacetime of all spherical gravitating bodies (not necessarily static) is described by the Schwarzschild metric.

The proof is simple. See, e.g., Misner et al. [3].

\subsection{Spherical Stars}

Generally, we assume that $\rho$ and $p$ satisfy an equation of state, e.g., in the form given by (C.25) and (C.26), and are generally functions of local $T$ and $\mu$ which in turn depend on $\xi$ through the Tolman equations (C.10). Numerical integration of (2.8) and (2.9) from $r=0$ to some radius $R$ with the boundary conditions

$$
\xi(R)=\left(1-\frac{2 \mathscr{M}(R)}{R}\right)^{1 / 2} ; \quad \mathscr{M}(0)=0
$$

is rather straightforward and, as a result, a nontrivial spherical distribution of matter is obtained. The boundary is usually naturally provided at the radius $R$ where $\rho=p=0$. In the case when the $\rho=p=0$ point does not exist, one must integrate up to infinity or up to a chosen cutoff radius.

It is often more convenient (e.g., if the equation of state is given in the form $p=p(\rho)$ ) to express the field equations in the Tolman-Oppenheimer-Volkoff (TOV) form

$$
\begin{gathered}
\frac{d p}{d r}=-(p+\rho) \frac{\mathscr{M}+4 \pi r^{3} p}{r(r-2 \mathscr{M})}, \\
\frac{d \mathscr{M}}{d r}=4 \pi r^{2} \rho .
\end{gathered}
$$

Here, equation (2.18) is obtained from (2.8) with the help of the equation of hydrostatic equilibrium (B.14) which may be written as

$$
\frac{d \ln \xi}{d r}=-\frac{1}{\rho+p} \frac{d p}{d r}
$$

The set of equations (2.18)-(2.20) is called the TOV equations.

\subsection{Newtonian Limit}

General relativity reduces to Newtonian theory in the limit of weak gravity and low velocities. The Newtonian limit is achieved by the approximation $\xi=e^{\varphi} \simeq 1+\varphi, \mathscr{M} / r \ll 1$, and $p \ll \rho$. In this limit, the two equations, (2.18) and (2.19), can be combined to give one 2nd-order differential equation

$$
\frac{1}{r^{2}} \frac{d}{d r} \frac{r^{2}}{\rho} \frac{d p}{d r}=-4 \pi \rho
$$

In this approximation, the relativistic energy density $\rho$ is just the rest mass density

$$
\rho=m n \text {. }
$$




\subsubsection{Polytropes}

If the equation of state is in the polytropic form (C.32), the equilibrium configurations are called polytropes. Owing to (2.22) we can rewrite the equation of state as

$$
p=K \rho^{\Gamma}
$$

where

$$
K=\frac{\mathscr{K}}{m^{\Gamma}}
$$

It is convenient to write

$$
\Gamma=1+1 / n
$$

where $n$ is called the polytropic index. Then the Newtonian equation (2.21) can be reduced to a simple form by writing

$$
\begin{gathered}
\rho=\rho_{c} \theta ; \quad r=\alpha z, \\
\alpha=\left[\frac{(n+1) K \rho_{c}^{1 / n-1}}{4 \pi}\right]^{1 / 2},
\end{gathered}
$$

where $\rho_{c}$ is the central density. Then

$$
\frac{1}{z^{2}} \frac{d}{d z} z^{2} \frac{d \theta}{d z}=-\theta^{n}
$$

This is the Lane-Emden equation for the structure of a polytropic index $n$. This equation can be numerically integrated starting from the center with the initial conditions

$$
\theta(0)=1 ; \quad \theta^{\prime}(0)=0
$$

For $n<5(\Gamma>6 / 5)$, the solutions decrease monotonically and have a zero at a finite value $z=z_{1}$; $\theta\left(z_{1}\right)=0$. This point corresponds to the surface of the star, where $\rho=p=0$. The radius is

$$
R=\alpha z_{1}=\left[\frac{(n+1) K}{4 \pi}\right]^{1 / 2} \rho_{c}^{(1-n) / 2 n} z_{1}
$$

while the mass is

$$
\begin{aligned}
M & =\int 4 \pi r^{2} \rho d r \\
& =4 \pi \alpha^{3} \rho_{c} \int_{0}^{z_{1}} z^{2} \theta^{n} d z \\
& =-4 \pi \alpha^{3} \rho_{c} \int_{0}^{z_{1}} \frac{d}{d z} z^{2} \frac{d \theta}{d z} d z \\
& =4 \pi\left[\frac{(n+1) K}{4 \pi}\right]^{3 / 2} \rho_{c}^{(3-n) / 2 n} z_{1}^{2}\left|\theta_{1}^{\prime}\right|,
\end{aligned}
$$

where the absolute value $\left|\theta_{1}^{\prime}\right|$ has been introduced because $\theta_{1}^{\prime} \equiv \theta^{\prime}\left(z_{1}\right)$ is negative. Eliminating $\rho_{c}$ between (2.30) and (2.31) gives the mass-radius relation for polytropes [4]:

$$
M=4 \pi R^{(3-n) /(1-n)}\left[\frac{(n+1) K}{4 \pi}\right]^{n /(n-1)} z_{1}^{(3-n) /(1-n)} z_{1}^{2}\left|\theta_{1}^{\prime}\right| .
$$


The solutions we are particularly interested in are

$$
\begin{gathered}
\Gamma=5 / 3, \quad n=3 / 2, \quad z_{1}=3.65375, \quad z_{1}^{2}\left|\theta_{1}^{\prime}\right|=2.71406, \\
\Gamma=4 / 3, \quad n=3, \quad z_{1}=6.89685, \quad z_{1}^{2}\left|\theta_{1}^{\prime}\right|=2.01824,
\end{gathered}
$$

where the values $5 / 3$ and $4 / 3$ of $\Gamma$ correspond to the nonrelativistic and ultrarelativistic regimes, respectively (see appendices C.4 and C.5).

\section{Compact Astrophysical Objects}

Traditionally, compact astrophysical objects represent the final stages of stellar evolution: white dwarfs (WD), neutron stars (NS), and black holes (BH). They differ from normal stars in two basic ways.

First, since they do not burn nuclear fuel, they cannot support themselves against gravitational collapse by generating thermal pressure. Instead, either they are prevented from collapsing by the degeneracy pressure (WDs and NSs) or they are completely collapsed (BHs). With the exception of the spontaneously radiating "mini" BHs with masses less than $10^{12} \mathrm{~kg}$ and radii smaller than a fermi, all these objects are essentially static over the lifetime of the universe.

The second characteristic distinguishing compact objects from normal stars is their exceedingly small size. Relative to normal stars of comparable mass, compact objects have much smaller radii and hence, much stronger surface gravity.

\begin{tabular}{|l|cccc|}
\hline Object & $\begin{array}{c}\text { Mass } \\
M\end{array}$ & $\begin{array}{c}\text { Radius } \\
R\end{array}$ & $\begin{array}{c}\text { Mean Density } \\
\mathrm{g} \mathrm{cm}^{-3}\end{array}$ & $\begin{array}{c}\text { Surface Potential } \\
\mathrm{M} / \mathrm{R}\end{array}$ \\
\hline Sun & $M_{\odot}$ & $R_{\odot}$ & 1 & $10^{-6}$ \\
White dwarf & $\sim M_{\odot}$ & $\sim 10^{-2} R_{\odot}$ & $\lesssim 10^{7}$ & $\sim 10^{-4}$ \\
Neutron star & $\sim 1-3 M_{\odot}$ & $\sim 10^{-5} R_{\odot}$ & $\sim 10^{15}$ & $\sim 10^{-1}$ \\
Black hole & Arbitrary & $2 M$ & $\sim M / R^{3}$ & $\sim 1$ \\
\hline
\end{tabular}

$M_{\odot}=1.989 \times 10^{30} \mathrm{~kg} ; R_{\odot}=6.9599 \times 10^{5} \mathrm{~km}$

\subsection{White Dwarfs}

White dwarfs are stars that no longer burn their nuclear fuel and their gravitational collapse is supported by the pressure of degenerate electrons. Their mass and radius are about $1 M_{\odot}$ and $5000 \mathrm{~km}$, respectively.

\subsubsection{Equation of State}

We assume that the interior of a WD is almost completely ionized plasma at a temperature $T \ll m_{e}$. Hence, the electrons are assumed to be degenerate. The density $\rho$ of a WD is basically the density of barionic matter (neutrons end protons). The pressure is dominated by the pressure of electrons. This may be seen as follows. In the nonrelativistic regime the pressure roughly equals the average kinetic energy (see equation (C.14)). Owing to the momentum conservation, the electron and proton average momenta are equal and hence, their average kinetic energies in the 
nonrelativistic regime are inverse proportional to their masses. Hence, the pressure of electrons is roughly by a factor of $m_{n} / m_{e} \simeq 2000$ larger than the pressure due to nucleons.

To determine the equation of state, first assume that the electrons are nonrelativistic. The density is related to the number density of electrons as

$$
\rho=\eta_{e} m_{n} n_{e}
$$

where the factor $\eta_{e}$, to a good approximation, equals the number of nucleons per free electron, i.e., $\eta_{e}=A / Z$. For example, for completely ionized pure ${ }^{12} \mathrm{C}, \eta_{e}=2$. The number of electrons is given by (C.24), with $m=m_{e}$. Hence,

$$
\rho=\frac{1}{3 \pi^{2}} \eta_{e} m_{B}\left(m_{e} X\right)^{3},
$$

where $m_{e} X=q_{F}=\sqrt{\mu^{2}-m_{e}^{2}}$ is the Fermi momentum of the electrons. We could now use the relativistic expression (C.26) for the pressure together with (3.2) and numerically solve the TOV equations to find the entire range of white-dwarf solutions.

However, it is instructive to consider the nonrelativistic and extreme relativistic regimes separately because, in these cases, the equation of state takes a polytropic form. The pressure in the two regimes is given by (2.23) with $\Gamma=5 / 3$ for the nonrelativistic and $\Gamma=4 / 3$ for the extreme relativistic regime. Using this and the solutions for the polytropes (2.30)-(2.34) we find the radius and the mass of the WD:

- Low-density (nonrelativistic) regime.

$$
\begin{gathered}
\Gamma=\frac{5}{3} ; \quad K=\frac{3^{2 / 3} \pi^{4 / 3}}{5 m_{e} m_{n}^{5 / 3} \eta_{e}^{5 / 3}} \\
R=1.122 \times 10^{4}\left(\frac{\rho_{c}}{10^{6} \mathrm{~g} \mathrm{~cm}^{-3}}\right)^{-1 / 6}\left(\frac{\eta_{e}}{2}\right)^{-5 / 6} \mathrm{~km}, \\
M=0.4964\left(\frac{\rho_{c}}{10^{6} \mathrm{gcm}^{-3}}\right)^{1 / 2}\left(\frac{\eta_{e}}{2}\right)^{-5 / 2} M_{\odot}, \\
=0.7011\left(\frac{R}{10^{4} \mathrm{~km}}\right)^{-3}\left(\frac{\eta_{e}}{2}\right)^{-5} M_{\odot}
\end{gathered}
$$

- High-density (ultrarelativistic) regime.

$$
\begin{gathered}
\Gamma=\frac{4}{3} ; \quad K=\frac{3^{1 / 3} \pi^{2 / 3}}{4 m_{n}^{4 / 3} \eta_{e}^{4 / 3}}, \\
R=3.347 \times 10^{4}\left(\frac{\rho_{c}}{10^{6} \mathrm{gcm}^{-3}}\right)^{-1 / 3}\left(\frac{\eta_{e}}{2}\right)^{-2 / 3} \mathrm{~km}, \\
M=1.457\left(\frac{\eta_{e}}{2}\right)^{2} M_{\odot} .
\end{gathered}
$$


Note that $M$ is independent of $\rho_{c}$ in the extreme relativistic limit. We conclude that as $\rho_{c} \rightarrow \infty$, the electrons become more and more relativistic and the mass asymptotically approaches the value (3.8) as $R \rightarrow 0$. This mass limit is called the Chandrasekhar limit, and represents the maximum possible mass of a WD [11].

Of course, the limiting radius is not zero. The integration of the TOV equations using the exact degenerate Fermi gas equation of state would also give the limiting value of $R$ of the order of

$$
R_{\mathrm{Ch}} \sim \frac{m_{\mathrm{Pl}}}{m_{n} m_{e}} \sim 5 \times 10^{3} \mathrm{~km} .
$$

\subsection{Neutron Stars}

If the mass of the collapsing star is larger than the Chandrasekhar limit, the degeneracy pressure of the electrons can no longer support the gravitational attraction and the collapse does not stop. As the density increases, the Fermi energy $E_{\mathrm{F}}=\mu$ of the electrons increases according to (3.2).

At a density of about $2 \times 10^{7} \mathrm{~g} \mathrm{~cm}^{-3}$, the Fermi energy of the electrons has risen to $m_{n}-m_{p}=$ $1.29 \mathrm{MeV}$ where electrons can now be absorbed by protons through the inverse $\beta$ decay

$$
e^{-}+p \rightarrow n+v_{e}
$$

This reaction cannot come to equilibrium with the reverse reaction

$$
n+v_{e} \rightarrow e^{-}+p
$$

because the neutrinos escape from the star and the normal $\beta$ decay cannot occur because all electron energy levels below $E=m_{n}-m_{p}$ are occupied when $E_{\mathrm{F}}>m_{n}-m_{p}$. At a density in the range $10^{7} \leq \rho \leq 4 \times 10^{11} \mathrm{~g} \mathrm{~cm}^{-3}$ the medium is a composition of separated nuclei in equilibrium with a relativistic electron gas. At $\rho \sim 4 \times 10^{11}$ the ratio $n / p$ reaches a critical level. Any further increase leads to a "neutron drip" - that is, a two-phase system in which electrons, nuclei, and free neutrons coexist. When the density exceeds about $4 \times 10^{12} \mathrm{~g} \mathrm{~cm}^{-3}$, more pressure is provided by neutrons than by electrons. The neutron gas so controls the situation; one can describe the medium as one vast nucleus with lower-than-normal nuclear density.

As the density reaches the normal nuclear density of about

$$
\rho_{\text {nucl }}=\frac{1 G e V}{(2 \mathrm{fm})^{3}}=2 \times 10^{14} \mathrm{gcm}^{-3},
$$

there is a phase transition in which nuclei dissolve. The resulting fluid consists mostly of neutrons with a small ( $\sim 5 \%$ ) fraction of electrons and protons. The pressure is dominated by the degenerate $\left(T=0, \mu_{n}>m_{n}\right)$ gas of neutrons. Can the degeneracy pressure of neutrons support the star against collapse?

Assuming that the Newtonian calculations are still valid at a density not very much higher than $\rho_{\text {nucl }}$, we can use the high-density results obtained for WDs but with $m_{e} \rightarrow m_{n}$. The maximal mass remains the same as in (3.9) but the value of the critical $R$ is of the order of

$$
R \sim \frac{m_{\mathrm{Pl}}}{m_{n}^{2}} \sim 2.5 \mathrm{~km}
$$




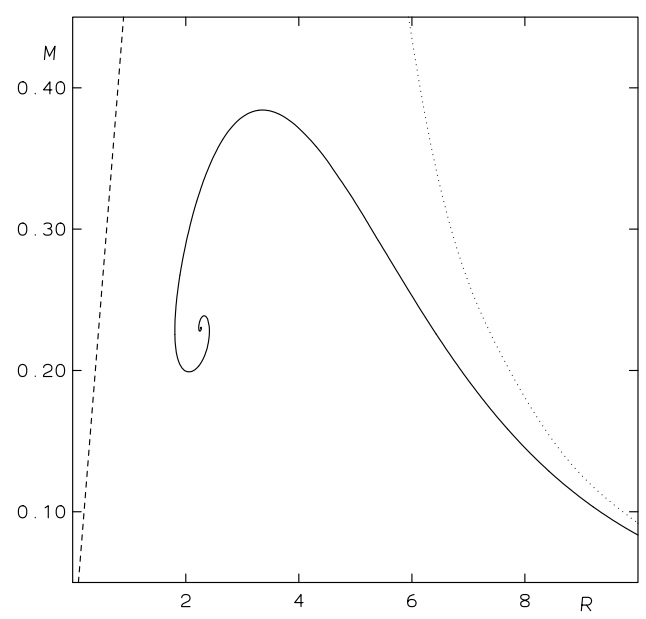

Figure 1: Mass versus radius for fermion stars at zero temperature in the general-relativistic framework (solid line) compared with the corresponding Newtonian approximation (dotted line). The dashed line is the Schwarzschild BH limit $M=R / 2$.

which is close to the Schwarzschild radius of the sun, so the neglect of GR effects is not justified.

We have to solve the TOV equations with the equation of states of nuclear matter. The first numerical calculation was performed by Oppenheimer and Volkoff [12] who used the relativistic degenerate Fermi gas described by (C.24)-(C.26). In Fig. 1 we plot the mass of the NS as a function of the radius $R$. The maximum of the curve corresponds to the OV limit. The limiting values are [13]

$$
\begin{gathered}
R_{\mathrm{OV}}=3.357 \frac{m_{\mathrm{Pl}}}{m^{2}}\left(\frac{2}{g}\right)^{1 / 2}=9.6\left(\frac{1 \mathrm{GeV}}{m^{2}}\right)^{2}\left(\frac{2}{g} \mathrm{~km}\right)^{1 / 2}, \\
M_{\mathrm{OV}}=0.38426 \frac{m_{\mathrm{Pl}}^{3}}{m^{2}}\left(\frac{2}{g}\right)^{1 / 2}=0.7\left(\frac{1 \mathrm{GeV}}{m^{2}}\right)^{2}\left(\frac{2}{g}\right)^{1 / 2} M_{\odot},
\end{gathered}
$$

where $g$ is the fermion degeneracy factor. This limit is reached when the central density becomes $\rho_{c}=5 \times 10^{15} \mathrm{~g} \mathrm{~cm}^{-3}$ with $g=2$ for neutrons. The part of the curve left from the maximum represents unstable configurations that curl up around the point corresponding to the infinite centraldensity limit.

However, the degenerate neutron gas equation of state is not realistic because at such large densities the effects of nuclear forces must be included. More realistic equations of state predict the maximum NS mass in the range 1.5 - 2.7 $M_{\odot}$. Hence, the maximal mass is rather sensitive to the not very well-known equation of state for nuclear matter. Since the density inside the star varies from very large central values to zero at the surface, the equation of state is actually rather complicated as the star may contain different phases of nuclear matter.

The possibility to identify some compact objects as BHs relies in part on being able to state categorically that the observed object has a mass larger than the maximum allowed mass of a stable 
NS. It turns out that it is possible to set an upper limit to the NS mass based on rather general assumptions (Rhodes and Ruffini [14]):

1. The TOV equation determines the equilibrium structure.

2. The equation of state satisfies the local stability condition

$$
c_{s}^{2} \equiv \frac{\partial p}{\partial \rho} \geq 0
$$

that is, the speed of sound is real. If this condition were violated, small elements of matter would spontaneously collapse.

3. The equation of state satisfies the causality condition

$$
c_{s}^{2} \leq 1
$$

that is, the speed of sound is less than the speed of light.

4. The equation of state below some "matching density" $\rho_{0}$ is known.

Rhodes and Ruffini performed a variational calculation to determine which equation of state above $\rho_{0}$ maximizes the mass. Then, the numerical integration of the TOV equations for a chosen equation of state below $\rho_{0}$ gives

$$
M_{\max } \simeq 3.2\left(\frac{\rho_{0}}{4.6 \times 10^{14} \mathrm{~g} \mathrm{~cm}^{-3}}\right)^{-1 / 2} M_{\odot} .
$$

A semianalytic treatment of Nauenberg and Chapline [15] with similar assumptions about the equation of state gives

$$
M_{\max } \simeq 3.6 M_{\odot} .
$$

Abandoning the causality constraint still leads to a severe mass limit, assuming general relativity to be valid. One finds (see, e.g., Hartle and Sabbadini [16])

$$
M_{\max } \simeq 5.2 M_{\odot} .
$$

In their estimate, Rhodes and Ruffini took the the so-called Harrison-Wheeler equation of state which accurately describes the nuclear matter densities below the neutron drip. In fact, it turns out that the upper mass limit is not very sensitive to the equation of state used below $\rho_{0}$. It is important to note that even if a new physics exists at subnuclear and subquark level (preons, pre-preons etc.; see, e.g., a black-hole sceptical paper [17]), it is reasonable to expect that the equation of state will still satisfy the above conditions and hence, the above limits cannot be significantly altered.

\subsection{Black Holes}

What happens if the mass of the collapsing star is larger than the maximal allowed NS mass? In such a case, there is nothing to prevent the star from further collapse ending in a $\mathrm{BH}^{2}$.

\footnotetext{
${ }^{2}$ It has been recently proposed that loop quantum gravity effects stop the collapse to a singularity by a bounce of the infalling matter [18]
} 
Astrophysical BHs are macroscopic objects with masses ranging from several $M_{\odot}$ (X-ray binaries) to $10^{6}-10^{9.5} M_{\odot}$ (in galactic nuclei). Being so massive, these BHs are described completely by classical general relativity. As such, each $\mathrm{BH}$ is characterized by just three numbers: mass $M$, spin parameter $a$, defined such that the angular momentum of the $\mathrm{BH}$ is $J=a M$, and electric charge $Q[3,4,19]$ (see also J. Zanelli's lectures at this School). Actually, an astrophysical BH is not likely to have any significant electric charge because it will usually be rapidly neutralized by surrounding plasma. Therefore, the BH can be fully characterized by measuring just two parameters, $M$ and $a$, of which the latter is constrained to lie in the range from 0 (nonrotating $\mathrm{BH}$ ) to $M$ (maximally-rotating $\mathrm{BH}$ ).

\subsubsection{Spherical Collapse}

A useful toy model that illustrates the collapse is a self-gravitating spherically symmetric ball of dust (i.e., zero pressure fluid). Birkhoff's theorem (section 2.2.1) implies that the metric outside the star is Schwarzschild (section 2.2, equation (2.15)). This is valid outside the star but also, by continuity of the metric, on the surface. If $r=R(t)$ on the surface, we have

$$
d s^{2}=\left[\left(1-\frac{2 M}{R}\right)-\left(1-\frac{2 M}{R}\right)^{-1}\left(\frac{d R}{d t}\right)^{2}\right] d t^{2}-R^{2}\left(d \theta^{2}+\sin ^{2} \theta d \phi^{2}\right)
$$

Zero pressure and spherical symmetry imply that a point on the surface follows a radial timelike geodesic, $d \theta=d \phi=0$ and $d s^{2}=d \tau^{2}>0$, so

$$
1=\left[\left(1-\frac{2 M}{R}\right)-\left(1-\frac{2 M}{R}\right)^{-1}\left(\frac{d R}{d t}\right)^{2}\right] \dot{t}^{2},
$$

where

$$
\dot{t}=\frac{d t}{d \tau}
$$

Since $\xi=\partial / \partial t$ is a Killing vector, by Proposition 1 in appendix A.5 the energy per unit mass

$$
E=\xi_{\mu} \frac{d x^{\mu}}{d \tau}=g_{00} \dot{t}=\left(1-\frac{2 M}{R}\right) \dot{t}
$$

is a constant of motion. Note that $E>0$ for timelike and null geodesics as long as $\xi^{\mu}$ is timelike, i.e., as long as $R>2 M$. The quantity $E$ is constant along the geodesics and $E<1$ for gravitationally bound particles. Using (3.24) in (3.22) gives

$$
\left(\frac{d R}{d t}\right)^{2}=\frac{1}{E^{2}}\left(1-\frac{2 M}{R}\right)^{2}\left(\frac{2 M}{R}-1+E^{2}\right) .
$$

We plot this function in Fig. 2. The surface radial velocity $d R / d t$ as a function of $R$ has a zero at $R=R_{\max }$ and a minimum at $R=2 M$. We consider the collapse to begin at $R=R_{\max }$ with zero velocity. The radius $R$ then decreases and approaches $R=2 M$ asymptotically as $t \rightarrow \infty$. This may be seen by integrating

$$
t=E \int_{R_{\max }}^{R_{\min }} d R(1-2 M / R)^{-1}\left(2 M / R-1+E^{2}\right)^{-1 / 2},
$$




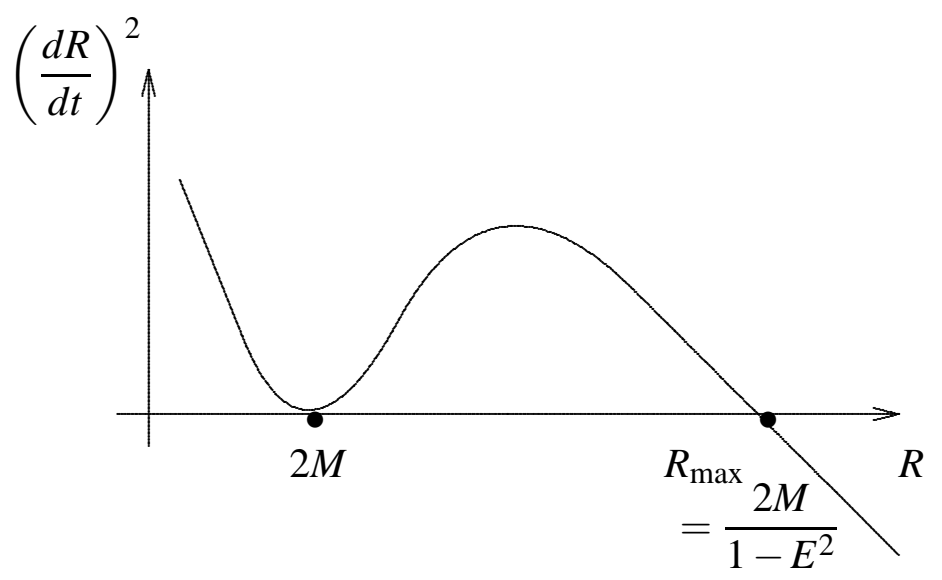

Figure 2: The surface radial velocity squared versus $R$ for a spherically symmetric collapse of a ball of dust (figure from [7]).

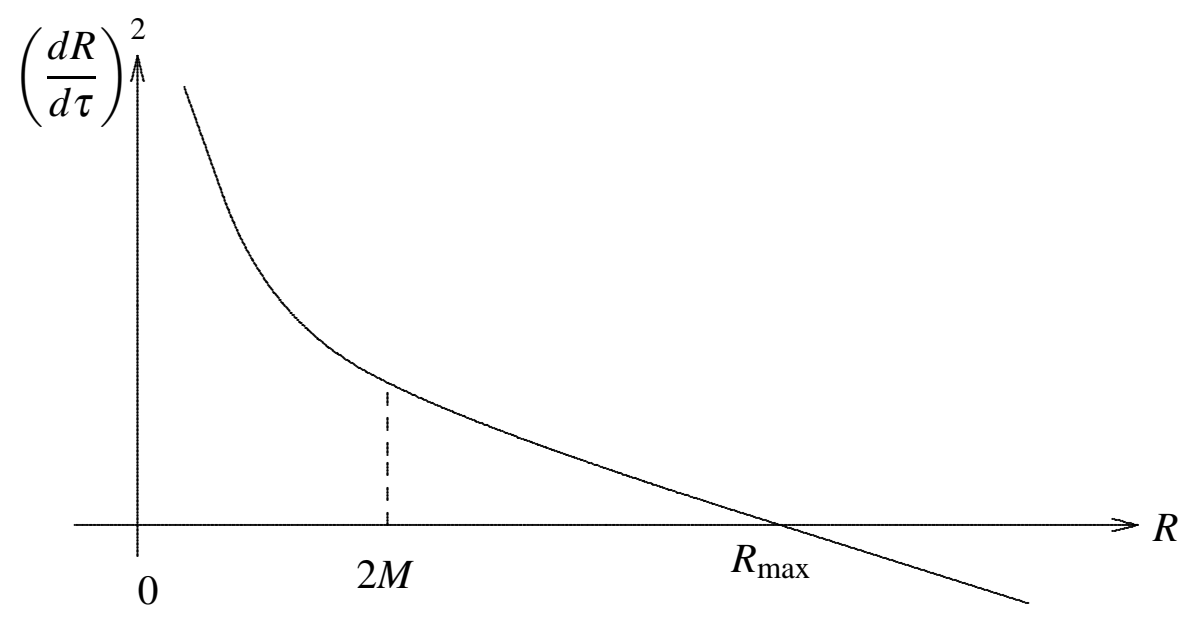

Figure 3: The surface radial velocity as seen by the observer on the surface (figure from [7]).

where $R_{\min }>2 M$. Obviously $t \rightarrow \infty$ as $R_{\min } \rightarrow 2 M$. So an observer "sees" the star contract at most to $R=2 M$ but no further.

However, from the point of view of an observer on the surface of the star, the relevant time variable is the proper time along a radial geodesic, so we use

$$
\frac{d}{d t}=\frac{1}{\dot{t}} \frac{d}{d \tau}=\frac{1}{E}\left(1-\frac{2 M}{R}\right) \frac{d}{d \tau}
$$

to rewrite (3.25) as

$$
\left(\frac{d R}{d \tau}\right)^{2}=\left(\frac{2 M}{R}-1+E^{2}\right)=\left(1-E^{2}\right)\left(\frac{R_{\max }}{R}-1\right)
$$

The star surface falls from $R=R_{\max }$ through $R=2 M$ in finite proper time. In fact, it reaches the 
$R=0$ singularity in the proper time

$$
\tau=\frac{\pi M}{(1-E)^{3 / 2}}
$$

So, for an observer following radial geodesics, the star collapses to a singularity in a finite proper time of the order of $10^{-5} \mathrm{~s}\left(\sim R_{\mathrm{Sch}} / c=3 \mathrm{~km} / 300000 \mathrm{~km} \mathrm{~s}^{-1}\right)$.

Nothing special happens at $R=2 M$, which suggests that we investigate the spacetime near $R=2 M$ in coordinates adapted to infalling observers.

\subsubsection{Eddington-Finkelstein Coordinates}

A singularity of the metric is a point at which the determinant of either the metric tensor or of its inverse vanishes, or at which some elements of the metric or its inverse diverge. However, a singularity of the metric may be simply due to a failure of the coordinate system. A simple twodimensional example is the origin in plane polar coordinates. Such singularities, usually referred to as coordinate singularities, are removable by a coordinate transformation.

If no coordinate system exists for which the singularity is removable, then it is irremovable, i.e., a genuine singularity of spacetime. Any singularity for which some scalar constructed from the curvature tensor blows up as it is approached, is irremovable. Such singularities are called curvature singularities or true singularities. The singularity at $r=0$ in the Schwarzschild metric is an example.

We now show that the apparent singularity of the Schwarzschild metric at the Schwarzschild radius is removable. On radial null geodesics in Schwarzschild spacetime

$$
d t^{2}=\frac{1}{(1-2 M / r)^{2}} d r^{2} \equiv\left(d r^{*}\right)^{2}
$$

where

$$
r^{*}=r+2 M \ln \left|\frac{r-2 M}{2 M}\right|
$$

is the Regge-Wheeler or tortoise radial coordinate. As $r$ ranges from $2 M$ to $\infty, r^{*}$ ranges from $-\infty$ to $\infty$. Thus

$$
d\left(t \pm r^{*}\right)=0
$$

on radial null geodesics. Now, define a new time coordinate (ingoing radial null coordinate) $v$ by

$$
v=t+r^{*}, \quad-\infty<v<\infty
$$

and rewrite the Schwarzschild metric in ingoing Eddington-Finkelstein coordinates [20] $v, r, \theta$, and $\phi$ :

$$
\begin{aligned}
d s^{2} & =\left(1-\frac{2 M}{r}\right)\left(d t^{2}-d r^{* 2}\right)-r^{2} d \Omega^{2} \\
& =\left(1-\frac{2 M}{r}\right) d v^{2}-2 d r d v-r^{2} d \Omega^{2}
\end{aligned}
$$

This metric is initially defined for $r>2 M$ because the relation $v=t+r^{*}(r)$ between $v$ and $r$ is defined only for $r>2 M$, but it can now be analytically continued to all $r>0$. Because of 
the $d r d v$ cross-term, the metric in EF coordinates is nonsingular at $r=2 M$, so the singularity in Schwarzschild coordinates is really a coordinate singularity. There is nothing at $r=2 M$ to prevent the star from collapsing through $r=2 M$.

However, no future-directed timelike or null worldline can reach $r>2 M$ from $r \leq 2 M$. This may be seen as follows. When $r \leq 2 M$, we have

$$
2 d r d v=-d s^{2}-\left(\frac{2 M}{r}-1\right) d v^{2}-r^{2} d \Omega^{2} \leq 0 \text { when } d s^{2} \geq 0 .
$$

Hence, for all timelike or null worldlines, $d r d v \leq 0$. The null coordinate $d v>0$ for future-directed worldlines, so $d r \leq 0$ with equality when $r=2 M, d \Omega=0$ (i.e., ingoing radial null geodesics at $r=2 M)$.

Thus, no signal from the star's surface can escape to infinity once the surface has passed through $r=2 M$. The star has collapsed to a black hole. For the external observer, the surface never actually reaches $r=2 M$, but as $r \rightarrow 2 M$, the redshift of light leaving the surface increases exponentially fast [19] and the star effectively disappears from view within a time $\sim M / m_{\mathrm{Pl}}^{2} \simeq$ $5 \times 10^{-6} \mathrm{~s}$. The late time appearance is dominated by photons escaping from the unstable photon orbit at $r=3 M$.

From the point of view of an outside observer, a star collapsing to a black hole never appears to collapse, but rather freezes at the horizon. How then can it be said that the star collapses to a singularity, if it never appears to collapse even till the end of the Universe?

The star does in fact collapse inside the horizon, even though an outside observer sees the star freezing at the horizon. The freezing can be regarded as a light travel time effect. Space can be regarded as falling into the black hole, reaching the speed of light at the horizon, and exceeding the speed of light inside the horizon [21]. The photons that are exactly at the horizon and are pointed radially upwards stay there for ever, their outward motion through space at the speed of light being canceled by the inward flow of space at the speed of light. It follows that it takes an infinite time for light to travel from the horizon to the external observer. The star does actually collapse: it just takes an infinite time for the information that it has collapsed to get to the outside world!

\section{Rotating Black Holes}

The spacetime around a rotating black hole is described by the Kerr metric expressed in BoyerLindquist coordinates $[3,4,5]$

$$
\begin{aligned}
d s^{2}= & \left(1-\frac{2 M r}{\Sigma}\right) d t^{2}+\frac{4 a M r \sin ^{2} \theta}{\Sigma} d t d \phi \\
& -\left(\frac{\left(r^{2}+a^{2}\right)^{2}-\Delta a^{2} \sin ^{2} \theta}{\Sigma}\right) \sin ^{2} \theta d \phi^{2}-\frac{\Sigma}{\Delta} d r^{2}-\Sigma d \theta^{2},
\end{aligned}
$$

with

$$
\Delta=r^{2}-2 M r+a^{2}, \quad \Sigma=r^{2}+a^{2} \cos ^{2} \theta .
$$

The parameters $a$ and $M$ are related to the total angular momentum $J=a M$. The horizon occurs at those points where $\Delta=0$, i.e., at the roots of the quadratic equation $\Delta=0$

$$
r_{ \pm}=M \pm \sqrt{M^{2}-a^{2}}
$$


Note that $|a|$ must be less than $M$ for a black hole to exist. If $a$ exceeded $M$, one would have a gravitational field with a "naked" singularity, i.e., one not within an event horizon. A naked singularity is forbidden by the so called cosmic censor conjecture (for details, see Wald [5] and references therein). A black hole with $|a|=M$ is called a maximally rotating black hole.

\section{Remarks}

- When $a=0$, the Kerr solution reduces to the Schwarzschild solution.

- Taking $\phi \rightarrow-\phi$ effectively changes the sign of $a$, so we may choose $a \geq 0$ without loss of generality.

\subsection{Geodesic Motion}

A straightforward approach to finding all geodesics is to integrate the geodesic equation (A.12) directly. However, it is often more economic to simplify the problem by symmetry considerations. The Kerr spacetime is stationary and axially symmetric so there exist two Killing vectors $\xi$ and $\psi$ which by Proposition 1 (appendix A.5) yield a conserved energy $E$ and an angular momentum $L$ (per unit mass) along geodesics

$$
\begin{gathered}
E=u^{\mu} \xi_{\mu}=\left(1-\frac{2 M r}{\Sigma}\right) \dot{t}+\frac{2 M a r \sin ^{2} \theta}{\Sigma} \dot{\phi}, \\
L=-u^{\mu} \psi_{\mu}=-\frac{2 M a r \sin ^{2} \theta}{\Sigma} \dot{t}+\left(\frac{\left(r^{2}+a^{2}\right)^{2}-\Delta a^{2} \sin ^{2} \theta}{\Sigma}\right) \sin ^{2} \theta \dot{\phi},
\end{gathered}
$$

where $\dot{x}^{\mu}=u^{\mu}=d x^{\mu} / d \tau$. In addition we have

$$
g_{\mu v} \dot{x}^{\mu} \dot{x}^{v}=\kappa
$$

where $\kappa=1,0$, and -1 for timelike, null, and spacelike geodesics, respectively. One may use equations (4.4) and (4.5) to solve for $\dot{t}$ and $\dot{\phi}$ in terms of $E$ and $L$, and substituting the results into (4.6) one obtains a differential equation for $\dot{r}$. In the case of equatorial geodesics, $\theta=\pi / 2$, one finds

where

$$
\frac{1}{2} \dot{r}+V(r)=0
$$

$$
V=-\kappa \frac{M}{r}+\frac{L^{2}}{2 r^{2}}+\frac{1}{2}\left(\kappa-E^{2}\right)\left(1+\frac{a^{2}}{r^{2}}\right)-\frac{M}{r^{3}}(L-a E)^{2}
$$

Thus, the problem of obtaining the geodesics in the equatorial plane reduces to solving a problem of ordinary, nonrelativistic, one-dimensional motion in an effective potential. The calculations are relatively simple for circular orbits. Circular orbits occur where $\dot{r}=0$, which requires

$$
V=0, \quad \frac{\partial V}{\partial r}=0
$$

For $\kappa>0$, equations (4.9) have solutions $\tilde{E}(r)$ and $\tilde{L}(r)$ for all $r>r_{\mathrm{ph}}$ (for details, see [4, 22]), where $r>r_{\mathrm{ph}}$ is the radius of the photon circular orbit. 


\subsubsection{Photon Circular Orbit}

Photon circular orbits are possible only for particular radii $r_{\mathrm{ph}}$. The requirements (4.9) for null geodesics $(\kappa=0$ in (4.8)) yield a cubic equation in $\sqrt{r}$

$$
r^{2}-2 M \pm 2 a \sqrt{M r}=0
$$

with the solution [22]

$$
r_{\mathrm{ph}}=2 M\left\{1+\cos \left[\frac{2}{3} \cos ^{-1}(\mp a / M)\right]\right\} .
$$

For $a=0, r_{\mathrm{ph}}=3 M$, while for $a=M, r_{\mathrm{ph}}=M$ (corotating) or $r_{\mathrm{ph}}=4 M$ (counterrotating orbit). For $\kappa=0$, equations (4.9) have a solution

$$
L= \pm E \sqrt{3 r_{\mathrm{ph}}^{2}+a^{2}}
$$

The photon orbit is the innermost boundary of the circular orbits for particles, i.e., for timelike geodesics $(\kappa>0)$.

\subsubsection{Angular Velocity}

The angular velocity of an orbiting particle is defined as

$$
\Omega=\frac{u^{\phi}}{u^{0}}=\frac{\dot{\phi}}{\dot{t}} .
$$

Using the parameterization (B.9) for the velocity components, it may be easily shown (exercise) that $\Omega$ can be expressed as

$$
\Omega=-\frac{g_{0 \phi}+\lambda g_{00}}{g_{\phi \phi}+\lambda g_{0 \phi}}
$$

where

$$
\lambda=-\frac{L}{E}=-\frac{u_{\phi}}{u_{0}} .
$$

For equatorial circular orbits one finds [22]

$$
\Omega= \pm \frac{M^{1 / 2}}{r^{3 / 2} \pm a M^{1 / 2}}
$$

This is the general-relativistic form of Kepler's third law for equatorial circular orbits. In this case, the quantity $\Omega$ is called the Keplerian frequency.

\subsubsection{Innermost Stable Circular Orbit}

It is obvious that not all circular orbits will be stable because, in addition to (4.9), stability requires

$$
\frac{\partial^{2} V}{\partial r^{2}} \geq 0
$$

From (4.8) we obtain

$$
1-E^{2} \geq \frac{2 M}{3 r}
$$


Substituting the solution $\tilde{E}(r)$ for timelike geodesics, we obtain a quartic equation in $\sqrt{r}$ for the limiting case of equality. The solution $r_{\mathrm{is}}$ is the radius of the innermost stable circular orbit (ISCO) also referred to as the marginally stable orbit [22]

$$
r_{\text {is }}=M\left[3+Z_{2} \mp\left(3-Z_{1}\right)^{1 / 2}\left(3+Z_{1}+2 Z_{2}\right)^{1 / 2}\right],
$$

with

$$
\begin{aligned}
& Z_{1}=1+\left(1-\frac{a^{2}}{M^{2}}\right)^{1 / 3}\left[\left(1+\frac{a^{2}}{M^{2}}\right)^{1 / 3}+\left(1-\frac{a^{2}}{M^{2}}\right)^{1 / 3}\right] \\
& Z_{2}=\left(3 \frac{a^{2}}{M^{2}}+Z_{1}^{2}\right)^{1 / 2} .
\end{aligned}
$$

For $a=0, r_{\text {is }}=6 M$; for $a=M, r_{\text {is }}=M$ (corotating) $r_{\text {is }}=9 M$ (counterrotating). Obviously, compared with the photon circular orbit, the innermost stable circular orbit satisfies $r_{\text {is }} \geq r_{\mathrm{ph}}$.

A quantity of great interest for the potential efficiency of a BH accretion disk as an energy source is the binding energy of the ISCO. Defining the efficiency $\eta$ as the maximum binding energy per unit rest mass, from (4.18) (with $=$ ) one finds

$$
\eta \equiv 1-\tilde{E}_{\mathrm{is}}=1-\left(1-\frac{2 M}{3 r_{\text {is }}}\right)^{1 / 2}
$$

Plugging in the solution (4.19), one finds that the efficiency $\eta$ increases from $1-\sqrt{8 / 9}$ (a=0) to $1-\sqrt{1 / 3}(\mathrm{a}=\mathrm{M})$ for corotating orbits, while it decreases from $1-\sqrt{8 / 9}(\mathrm{a}=0)$ to $1-\sqrt{25 / 27}$ $(\mathrm{a}=\mathrm{M})$ for counterrotating orbits. The maximum binding energy for a maximally rotating $\mathrm{BH}$ is $42.3 \%$ of the rest-mass! This is the amount of energy that is released by matter spiraling in toward the $\mathrm{BH}$ through a succession of almost circular equatorial orbits. A negligible amount of energy is released during the final plunge from $r_{\text {is }}$ into the $\mathrm{BH}$.

\subsection{Ergosphere}

A curious property of rotating $\mathrm{BHs}$ is that there exist particle trajectories (i.e., timelike geodesics) with negative energies. The energy defined by (4.4) can be negative only if the time translation Killing vector $\xi$ is spacelike. The vector $\xi$ is normally timelike at $\infty$ and in Schwarzschild spacetime it is timelike everywhere outside the horizon. However, in Kerr spacetime it need not be timelike everywhere outside the horizon because

$$
\xi^{\mu} \xi_{\mu}=g_{00}=\left(1-\frac{2 M r}{r^{2}+a^{2} \cos ^{2} \theta}\right)
$$

so $\xi$ is timelike only if

$$
r^{2}+a^{2} \cos ^{2} \theta-2 M r>0
$$

This implies

$$
\begin{aligned}
& r<M-\sqrt{M^{2}-a^{2} \cos ^{2} \theta}, \\
& r>M+\sqrt{M^{2}-a^{2} \cos ^{2} \theta} .
\end{aligned}
$$


The inner boundary of this region is not physically relevant as it is beyond the horizon. The outer boundary, i.e.,the hypersurface

$$
r=M+\sqrt{M^{2}-a^{2} \cos ^{2} \theta}
$$

is called the ergosphere or the stationary limit surface. The ergosphere intersects the event horizon at $\theta=0, \pi$, but it lies outside the horizon for other values of $\theta$. Thus, $\xi$ can become spacelike in a region outside the event horizon. This region is called the ergoregion. Hence, a particle trajectory inside the ergosphere may have negative energy!

\subsubsection{Penrose Process}

In 1969 Penrose exploited this property of Kerr BHs to design a mechanism for the extraction of energy from a BH [23]. The mechanism proposed by Penrose can be understood as follows. Suppose that a particle approaches a Kerr BH along a geodesic. If $q^{\mu}$ is its 4-momentum, we can identify the constant of motion

$$
E=q^{\mu} \xi_{\mu}
$$

as its energy (see section A.5). The trajectory is chosen so that it penetrates the ergosphere. Now suppose that the particle decays into two others, one of which falls into the hole with energy $E_{\text {in }}$, while the other escapes to $\infty$ with energy $E_{\text {out }}$. By conservation of energy

$$
E_{\text {out }}=E-E_{\text {in }} \text {. }
$$

Normally, $E_{\text {in }}>0$, so $E_{\text {out }}<E$, but $E_{\text {in }}=q_{\text {in }}^{\mu} \xi_{\mu}$ is not necessarily positive in the ergoregion because $\xi$ may be spacelike there. Thus, if the decay takes place in the ergoregion, we may have $E_{\text {out }}>E$, so energy has been extracted from the black hole.

The energy extraction by the Penrose process is limited by the area theorem of $\mathrm{BH}$ mechanics, which states that the surface area of the $\mathrm{BH}$ horizon never decreases [24]. The area of the horizon is

$$
A=\int \sqrt{\operatorname{det} g} d \theta d \phi=8 \pi M\left(M+\sqrt{M^{2}-a^{2}}\right),
$$

where det $g=g_{\theta \theta} g_{\phi \phi}$ is the determinant of the metric on the horizon surface. The surface metric is obtained from (4.1) by setting $d t=d r=0, r=r_{+}=M+\sqrt{M^{2}-a^{2}}$. The maximum energy extracted by the Penrose process is obtained if the BH spin reduces to zero, i.e., if the BH becomes Schwarzschild. A Schwarzschild BH with the same area will have a mass $M_{\text {irr }}$ (irreducible mass) which satisfies

$$
16 \pi M_{\mathrm{irr}}^{2}=8 \pi M\left(M+\sqrt{M^{2}-a^{2}}\right) .
$$

This gives $M_{\mathrm{irr}}^{2}=M / 2$ for a maximally rotating $\mathrm{BH}(a=M)$. Hence, the maximally extracted energy from a BH of mass $M$ is $M-M_{\text {irr }}=M(1-1 / \sqrt{2})$. This represents $\approx 29 \%$ of the mass energy of the $\mathrm{BH}$.

The Penrose process for extracting energy from a rotating $\mathrm{BH}$ requires particular conditions [22] which are very difficult to realize in nature. A more promising mechanism for extracting energy from a rotating BH is via magnetic fields (Znajek and Blandford [25, 26],) because magnetic fields are capable of connecting regions very close to the BH to regions farther out. Recent generalrelativistic magneto-hydrodynamics (MHD) simulations of a magnetized plasma in the ergosphere 
of a rotating BH (Koide et al. [27, 28, 29], Semenov et al. [30], de Villiers et al. [31], McKinney and Gammie [32]) show that, near the equatorial plane, the field lines are azimuthally twisted and the twist then propagates outward and transports the energy along the rotation axis. The process is dubbed the MHD Penrose process because of a close analogy between this mechanism [29] and Penrose's original idea [23].

\section{Stellar Mass BHs versus NSs}

Astrophysical observation of NSs and BHs is difficult because they are dark and very compact. Besides, a BH the mass of which is of the order of a few solar masses is not easily distingushed from a NS because of their similar properties. So far, NSs and stellar mass BH candidates have been identified only as the so-called $X$-ray binaries.

\subsection{X-ray Binaries}

An X-ray binary is an X-ray source with an optical companion, usually a normal star. Most of the Galactic X-ray sources are probably compact objects accreting gas from the companion star.

This interpretation of the observational data follows from these facts [4]:

1. The variability of X-ray emission on short timescales implies a small emitting region.

2. Many of the sources are positively confirmed to be in binary systems, with optical primaries orbiting optically invisible secondaries.

3. Mass accretion onto a compact object, especially a neutron star or a black hole, is an extremely efficient means of converting released gravitational potential energy into X-ray radiation.

In general, the list of possible Galactic X-ray source candidates includes all three kinds of compact objects: WDs, NSs, and BHs. But in special cases, the specific nature of the compact object can be identified.

\subsubsection{Binary X-ray Pulsars}

Binary X-ray sources displaying periodic variations are called binary X-ray pulsars. The pulse periods are observed in the range $0.5 \mathrm{~s} \sim P \curvearrowright 1000 \mathrm{~s}$. Those with short periods of about $1 \mathrm{~s}$ are normally identified with rotating NS.

The standard model explains X-ray emission as due to the conversion of the kinetic energy of the accreting matter (coming from the intense stellar wind of the companion optical star) into radiation, because of the interactions with the strong magnetic field ${ }^{3}$ of the neutron star, of the order of $10^{7}-10^{9} \mathrm{~T}$. The magnetic field of the compact object drives the accreted matter onto the magnetic polar caps, and if the magnetic field axis is not aligned with the spin axis, then the compact object acts as a "lighthouse", giving rise to pulsed emission when the beam (or the beams, according to the geometry) crosses our line of sight.

The reasons why WD and BHs cannot be pulsars are:

\footnotetext{
${ }^{3}$ Obtained from conservation of the magnetic flux during the process of collapse from a "normal" star $\left(B \sim 10^{-3}\right.$. $\left.10^{-2} \mathrm{~T}, R \sim 10^{6} \mathrm{~km}\right)$ to a neutron $\operatorname{star}(R \sim 10 \mathrm{~km})$
} 
- Rotating WD are excluded because they are too large and their density too low to explain such short periods.

- Rotating BH are excluded because they are axially symmetric and have no structure on which to attach a periodic emitter. Any mechanism depending on accretion would not be periodic to the observed precision. However, accretion disks round black holes could produce the so-called quasi periodic oscillations (see section 5.3)

\subsubsection{Mass Measurements}

The first important criteria that may be used to distinguish a BH from a NS are their masses. The fact that there exists a maximum mass of a compact relativistic star such as a NS of the order $\sim 3 M_{\odot}$ (see section. 3.2) allows the following simple criteria for identifying BH candidates [8]: If a compact astrophysical object has the mass larger than about $3 M_{\odot}$, then the object is very likely a black hole.

The most reliable means of determining astronomical masses are via Kepler's Third Law. Consider two spherical masses $M_{1}$ and $M_{2}$ in a circular orbit about their center of mass (CM). The separation of the two masses is $a$ and their distances from the CM are $a_{1}$ and $a_{2}$. Clearly, $a=a_{1}+a_{2}$ and $M_{1} a_{1}=M_{2} a_{2}$ by the definition of the CM. Any spectra emitted from, e.g., $M_{2}$ will be Doppler shifted, depending on the orbital velocity projection $v_{2}$ of $M_{2}$ along the line of sight:

$$
v_{2}=\frac{2 \pi}{P_{\text {orb }}} a_{2} \sin i
$$

where $P_{\text {orb }}$ is the orbital period and $i$ the inclination of the orbital plane to the line of sight. Thus, if the spectrum of $M_{1}$ shows periodic variations, then $P_{\text {orb }}$ and $v_{2}$ can be measured and hence one gets $a_{2} \sin i$. Alternatively, for X-ray pulses one can measure periodic variations in the time of arrival of pulses. The amplitude of these variations is simply the light travel time across the projected orbit that is, $a_{2} \sin i / c$.

Now, Kepler's Third Law states

$$
\frac{G\left(M_{1}+M_{2}\right)}{a^{3}}=\left(\frac{2 \pi}{P_{\text {orb }}}\right)^{2}
$$

Note that this is valid also for elliptical orbits in which case $a$ is the semimajor axis of the ellipse. Using this and

$$
a=\frac{M_{1}+M_{2}}{M_{1}} a_{2}
$$

we obtain

$$
f\left(M_{1}\right) \equiv \frac{\left(M_{1} \sin i\right)^{3}}{\left(M_{1}+M_{2}\right)^{2}}=\frac{P_{\mathrm{orb}} v_{2}^{3}}{2 \pi G} .
$$

The quantity $f$ is called the "mass function" and depends only on the observable quantities $P_{\text {orb }}$ and $v_{2}\left(\right.$ or $\left.a_{2} \sin i\right)$.

For several X-ray binaries, it has been possible to measure the mass functions $f_{O}=f\left(M_{O}\right)$ and $f_{X}=f\left(M_{X}\right)$ for both the optical companion and the X-ray source, respectively. The ratio $f_{O} / f_{X}$ gives th mass ratio

$$
q \equiv \frac{M_{O}}{M_{X}}=\left(\frac{f_{O}}{f_{X}}\right)^{1 / 3}
$$


and then from (5.4) we can write

$$
M_{X}=f_{X} \frac{(1+q)^{2}}{\sin ^{3} i}
$$

A unique value for $M_{X}$ still depends on knowing $\sin i$. In practice, the X-ray eclipse duration and/or variation in the optical light curve are used to set geometrical constraints on $\sin i$.

In this way a complete description of the binary system has been obtained for six eclipsing Xray pulsars with optical companions [4]. Their masses range from 1 to $2.3 M_{\odot}$, with error bars such that all the data fit in the range $1.2-1.6 M_{\odot}$, which is expected on the basis of current theoretical scenarios for NS formation. One of these X-ray pulsars is the famous Hercules X-1 neutron star discovered in 1972 [33] in the data of the first astronomy satellite, Uhuru launched by NASA off the coast of Kenya. ${ }^{4}$

\subsection{Black-Hole Binaries}

Black hole X-ray binaries, or short, $B H$ binaries are the binary X-ray sources with observed masses larger than $3 M_{\odot}$ with nonperiodic time variability. As of today there are a total of 20 confirmed BH binaries (Remillard and McClintock [1]) Their large masses makes them strong candidates for BHs. However, the mass estimates are reliable only for those for which the inclination angle $i$ is well known, which is not always the case. Fortunately, according to (5.6), $M>f(M)$. The mass function $f(M)$, which depends only on the two accurately measured quantities $v_{2}$ and $P_{\text {orb }}$, is a strict lower bound on $M$. Most of the 20 X-ray binaries have $f(M)$ itself larger than or of the order $3 M_{\odot}$. Therefore, these systems are excellent BH candidates, regardless of uncertainties in their inclinations and companion star masses.

\subsubsection{Cygnus X-1: A Black-Hole Candidate}

Cygnus X-1 is a typical BH binary discovered in 1972 (Webster and Murdin [34], Bolton [35]) as a first stellar-mass $\mathrm{BH}$ candidate. The $\mathrm{X}$-ray source $(\mathrm{X})$ of $\mathrm{Cyg} \mathrm{X}-1$ is variable on all timescales varying from ms to months and years. Recent observations show that Cyg X-1 periodically cycles through two accretion or spectral states: hard and low X-ray states [36]. The most dramatic variability is the 1-ms bursts, which set a maximum size for X of the order $R \curvearrowright 300 \mathrm{~km}$ and establish the object to be highly compact.

The optical companion star $(\mathrm{O})$ in the Cyg X-1 system is a typical supergiant star with a wellknown spectrum and the mass of at least $M_{O} \sim 8.5 M_{\odot}$ [4]. From the measurements of the orbital elements, $P_{\text {orb }}=5.6$ days and $a_{O} \sin i=(5.82 \pm 0.08) \times 10^{6} \mathrm{~km}$, one obtains the mass function of $\mathrm{X}, f_{X}=(0.252 \pm 0.010) M_{\odot}$. Setting $\sin i=1$ in (5.6), one obtains a minimum value for the mass

$$
M_{X} \gtrsim 3.3 M_{\odot}
$$

It is possible to set a convincing lower limit assuming nothing about the mass of $\mathrm{O}$ and using only the absence of a prominent $\mathrm{X}$-ray eclipse and the estimate of the radius of $\mathrm{O}$ [37]. The absence of eclipse implies

$$
\cos i \geq \frac{R}{a}
$$

\footnotetext{
4"Uhuru" means "freedom" in Swahili; the launch occurred on the anniversary of Kenya's independence.
} 
where $R$ is the radius of $\mathrm{O}$ and $a=a_{X}+a_{O}$ is the separation of the two objects. Using (5.3), we can write this as

$$
\cos i \geq \frac{R}{a_{O} \sin i} \frac{M_{X} \sin i}{M_{X}+M_{O}} .
$$

Hence,

$$
M_{X} \sin i \cos ^{2} i \geq \frac{f_{X} R^{2}}{\left(a_{O} \sin i\right)^{2}}
$$

The function $\sin x \cos x^{2}$ has a maximum value of $2 /(3 \sqrt{3})$, and so

$$
M_{X} \geq \frac{3 \sqrt{3} f_{X} R^{2}}{2\left(a_{O} \sin i\right)^{2}}
$$

Estimating $R$ from luminosity and the effective temperature, one finds [4]

$$
R=6.62 \times 10^{6}\left(\frac{d}{1 \mathrm{kpc}}\right) \mathrm{km} .
$$

From (5.12) with known $f_{X}$ and $a_{O} \sin i$ one finds

$$
M_{X} \geq 3.4\left(\frac{d}{2 \mathrm{kpc}}\right)^{2} M_{\odot} .
$$

The distance $d$ to Cyg X-1 is determined using two methods: a) from the assumed luminosity compared with the apparent luminosity of $\mathrm{O}$ and $\mathrm{b}$ ) from the absorption vs. distance curve calibrated from a large sample of stars in the same direction. The estimated distance for $\mathrm{O}$ is $d \simeq 2.5 \mathrm{kpc}$ with an absolute minimum of $2 \mathrm{kpc}$ in order to produce the observed absorption.

We can summarize the situation as follows: the lower limit (5.13) of $3.4 M_{\odot}$ is very solid. Adopting the more reasonable value of $d \simeq 2.5 \mathrm{kpc}$ increases this to $5.3 M_{\odot}$. Various other less rigorous but more realistic arguments, as well as more recent measurements of $R, d$, and $f_{X}$ give even larger values. The currently accepted mass range of Cyg X-1 is 6.8-13.3 $M_{\odot}[1]$.

\subsection{Spin Estimates}

Measuring the $\mathrm{BH}$ spin amounts to measuring the Kerr-spacetime parameter $a$. In contrast to $\mathrm{BH}$ mass estimates where Newtonian gravity applies, the spin of a $\mathrm{BH}$ or of any other rotating astrophysical object does not have any Newtonian effect on the surrounding objects. Only for relativistic orbits does spin have measurable effects. Therefore, to measure $a$, we need test particles orbiting very close to the innermost stable circular orbit (ISCO) (see section 4.1.3). Such test particles are provided by the accretion disk.

The gas in an accretion disk starts from large radii and spirals in through a sequence of nearly circular orbits as it viscously loses angular momentum. The main source of instability and loss of angular momentum are MHD effects, e.g., magnetorotational instability [38] and magnetoviscous instability [39]. When the gas reaches the ISCO, no more stable circular orbits are available, so the gas accelerates radially and free-falls into the BH. Thus, the ISCO serves effectively as the inner edge $^{5}$ of the accretion disk. A variety of observational methods have been proposed for estimating

\footnotetext{
${ }^{5}$ The inner edge may not be very pronounced; compare, e.g., the truncated disk model [40]
} 
the radius $r_{\text {in }}$ (spectral fitting method; relativistic iron line method), the Keplerian frequency $\Omega$ (quasiperiodic oscillations method), or the binding energy $\eta$ at $r_{\text {is }}$. Next, we briefly describe some of these methods. For more details and for references, see [8].

\section{Spectral Fitting}

When a $\mathrm{BH}$ has a large mass accretion rate $\dot{M}$, corresponding to an accretion luminosity $L_{\text {acc }}$ above a few per cent of $L_{\mathrm{Edd}}$ (see section 6.3.1), the accreting gas tends to radiate approximately as a blackbody. In this spectral state one can theoretically calculate the flux of radiation $F(r)$ emitted by the accretion disk, and hence obtain the effective temperature profile $T_{\text {eff }}(r) \equiv[F(r) / \sigma]^{1 / 4}$, where $\sigma$ is the Stefan-Boltzmann constant. If the disk emits as a true blackbody at each radius, it is a simple matter to calculate the total spectral luminosity $L_{v} d v$. By comparing this quantity with the spectral flux $F_{v} d v$ received at Earth, one obtains an estimate of $r_{\text {in }}^{2} \cos i / d^{2}$ (essentially the projected solid angle of the disk), where $i$ is the inclination angle and $d$ is the distance to the source. In a few BH binaries, sufficiently reliable estimates of $i, d$ and $M$ are available, and thus an estimate of $r_{\text {in }}$ is obtained. Identifying $r_{\text {in }}$ with $r_{\text {is }}$, one then obtains $a$.

A major weakness of this method is that a number of effects will cause the spectrum of an accretion disk to deviate from a blackbody. Besides, the method requires accurate estimates of $M$, $i$, and $d$. Therefore, spin estimates obtained by this method should be treated with caution.

\section{Quasiperiodic Oscillations}

For some BH binaries, the power spectrum of intensity variations shows one or two peaks (more like bumps in some cases) at frequencies of a few hundred Hz. The peaks are relatively broad, indicating that they do not correspond to coherent oscillations but rather to quasiperiodic oscillations (QPOs).

One possibility is that the QPO with the highest frequency in each BH binary corresponds to the circular Keplerian frequency of gas blobs at some characteristic radius; it is plausible that this radius corresponds to the inner edge of the disk. Using equations (4.16) and (4.19), one can express the Keplerian frequency of the ISCO as

$$
\Omega_{\mathrm{is}}=\frac{1}{M} F(a / M)
$$

where $F(x)$ is a known function of $x=a / M$. Assuming that $r_{\text {in }}=r_{\text {is }}$, one can use this method to estimate $a$ provided an estimate of $M$ is available. The method has been applied to a few $\mathrm{BH}$ binaries (for references, see [8]). Recently, there has been tentative evidence for QPOs with a period of 17 minutes in the infrared emission (Genzel et al. [41]) from Sgr A*, the supermassive BH in the Galactic Center. If the QPOs correspond to the Keplerian frequency at any radius $r>r_{\text {is }}$, then the $\mathrm{BH}$ must be rotating with $a>0.5$ [8]. A number of QPO frequencies in the X-ray flares from Sgr A* have been identified by Aschenbach et al. [42]. Their analysis reveals that the emission from the inner parts of the accretion disk is quite close to the BH horizon and they find $a \simeq 0.99$.

\section{Relativistic Iron line}


A strong broad spectral line in the X-ray spectrum of the active galactic nucleus (AGN) MCG6-30-15 was recently discovered [43]. They interpreted the line as fluorescent iron $\mathrm{K} \alpha$ emission from cool gas in the accretion disk. Similar broad lines were seen in a few other AGN and X-ray binaries. Whereas the rest energy of the iron line is $6.4 \mathrm{keV}$, the observed line extends from about 4 to $7 \mathrm{keV}$. This broadening is due to Doppler blue- and red-shifts as well as to the gravitational redshift.

The line width and its shape, among other factors, depend on the radius range over which the emission occurs and in particular on the position of the innermost radius of the disk which in turn depends on $a$ ( since $r_{\text {in }}=r_{\text {is }}$ ).

Given a system with a broad iron line, and assuming that the radiating gas follows Keplerian orbits with radii $r \geq r_{\text {is }}$, one can fit the shape of the line profile by adjusting $a, i$, and the emissivity function; the latter is usually modeled as a power law in radius, $r^{-\beta}$, motivated by the standard disk model [44]. The effect of $a$ is particularly dramatic. As the BH spin increases, the inner edge of the disk comes closer to the horizon and the velocity of the gas increases substantially. This gives a wider range of Doppler shifts, as well as a larger gravitational redshift. The detection of such extreme levels of broadening may be taken as a strong indication of a rapidly spinning $\mathrm{BH}$.

In the case of MCG-6-30-15, the data confirm that the emission comes from a relativistic disk and at least some of the data sets can be interpreted in terms of a rapidly spinning BH. Assuming that there is no emission from within the ISCO, Reynolds et al. [45] estimate $a>0.93$. Among BH binaries, the source GX 339-4 shows a broad iron line which seems to indicate $a>0.8$ (Miller et al. [46]).

In spite of some weaknesses, the method has the advantage that it requires no knowledge of the $\mathrm{BH}$ mass or of the distance, and it solves for the disk inclination $i$ using the same line data from which $a$ is estimated.

The variability of the line with time means that it will be challenging to make fundamental tests of gravity with this method. On the other hand, the variability could provide interesting opportunities to study disk dynamics and turbulence [47, 48] (movies courtesy P. Armitage).

\section{Supermassive Black Holes}

It is now widely accepted that quasars and active galactic nuclei are powered by accretion onto massive black holes [49, 50, 51]. Further, over the last few years there has been increasing evidence that massive dark objects may reside at the centers of most, if not all, galaxies [52, 53]. In several cases, the best explanation for the nature of these objects is that they are supermassive black holes, with masses ranging from $10^{6}$ to $10^{10}$ solar masses. Comprehensive lists of about 30 supermassive BHs at galactic centers may be found in $[2,54,55,56]$.

\subsection{Masses and Radii}

The main criterion for finding candidates for such black holes is the presence of a large mass within a small region. The mass and the size are estimated using mostly the following three methods: gas spectroscopy, maser interferometry, and measuring the motion of stars orbiting around the galactic nucleus. 


\subsubsection{Gas Spectroscopy}

An example of measurements via gas spectrography is given by the analysis of the Hubble Space Telescope observations of the radio galaxy M 87 [57, 58].

A spectral analysis shows the presence of a disklike structure of ionized gas in the innermost few arc seconds in the vicinity of the nucleus of M 87. The velocity of the gas measured by spectroscopy at a distance from the nucleus of the order $20 \mathrm{pc} \approx 6 \times 10^{14} \mathrm{~km}$, shows that the gas recedes from us on one side, and approaches us on the other, with a velocity difference of about $920 \mathrm{~km} \mathrm{~s}^{-1}$. This leads to a mass of the central object of $\sim 3 \times 10^{9} M_{\odot}$, and no form of matter can occupy such a small region except for a black hole. This is the most massive black hole ever observed.

\subsubsection{Maser Interferometry}

A clear and compelling evidence for black holes has recently been discovered in the radio regime: $\mathrm{H}_{2} \mathrm{O}$ masers orbiting compact supermassive central objects. The structure of accreting material around the nearby galaxy NGC $4258(d \simeq 6.4 \mathrm{Mpc})$ has been studied in detail [59, 60, 61]. with the aid of very long baseline interferometry (VLBI), which provides an angular resolution as fine as $200 \mu$ as (microarcseconds) at a wavelength of $1.3 \mathrm{~cm}$ and a spectral resolution of $0.1 \mathrm{~km} \mathrm{~s}^{-1}$ or less, radio interferometry measurements have shown that the gas follows circular orbits with a nearly perfect Keplerian velocity profile $\left(v \propto r^{-1 / 2}\right.$, see Fig. 1 in [8]). Furthermore, the acceleration of the gas has been measured and it too is consistent with Keplerian dynamics (Bragg et al. [62]). From these measurements it is inferred that there is a dark object with a mass of $3.5 \times 10^{7} M_{\odot}$ confined within $\sim 0.13 \mathrm{pc}=4 \times 10^{12} \mathrm{~km}$ of the center of NGC 4258. The case for this dark mass being a $\mathrm{BH}$ is quite strong.

\subsubsection{Virial Mass}

A simple method to obtain a mass estimate is based on the virial theorem. It uses the measured velocity dispersion of stars in the central region (ref. [6], p. 1007). For simplicity, we restrict attention to a spherical cluster of radius $R$ with $N$ stars, each of mass $m$, so the total mass of the bulge is $M=N m$.

The time-averaged kinetic and potential energies of stars in the galaxy's central region are related by the equation (ref. [6], p. 54-56)

$$
\frac{1}{2}\left\langle\frac{d^{2} I}{d t^{2}}\right\rangle-2\langle K\rangle=\langle U\rangle
$$

where $I$ is the region's moment of inertia. If the galaxy is in equilibrium, then $\left\langle d^{2} I / d t^{2}\right\rangle=0$, resulting in the usual statement of the virial theorem

$$
-2\langle K\rangle=\langle U\rangle
$$

Furthermore, for a large number of stars, the central bulge will look the same (in statistical sense) at any time, and the time averaging can be dropped. So for $N$ stars of equal mass, we find

$$
-\frac{m}{N} \sum v_{i}^{2}=\frac{U}{N}
$$


The average velocity squared is

$$
-\frac{1}{N} \sum v_{i}^{2}=\left\langle v^{2}\right\rangle=\left\langle v_{r}^{2}\right\rangle+\left\langle v_{\theta}^{2}\right\rangle+\left\langle v_{\phi}^{2}\right\rangle=3\left\langle v_{r}^{2}\right\rangle=3 \sigma_{r}^{2},
$$

where $\sigma_{r}$ is the dispersion in the radial velocity.

\section{Velocity dispersion}

For a region in space with a large number of stars $N, \sigma_{x}$ measures the spread in the $x$ component of the peculiar velocities of stars and is defined as

$$
\sigma_{x}=\frac{1}{N}\left[\sum_{i} v_{i x}^{2}\right]^{1 / 2}
$$

It is equal to the standard deviation of the velocity distribution in the special case when $\left\langle v_{x}\right\rangle=0$.

Using the (approximate) gravitational potential energy of a spherical distribution of the total mass (exercise)

$$
U=-\frac{3}{5} \frac{G M^{2}}{R}
$$

equations (6.2) and (6.3) yield

$$
M_{\text {virial }}=\frac{5 R \sigma_{r}^{2}}{G}
$$

where the mass obtained in this way is called the virial mass.

This equation can be used to estimate a virial mass for the central BH of M31 (Andromeda). The central radial velocity dispersion is measured to be approximately $240 \mathrm{~km} / \mathrm{s}$ within 0.2 as (arcseconds). Given the distance to Andromeda of $770 \mathrm{kpc}, 0.2$ as corresponds to $R \simeq 0.8 \mathrm{pc}$. This gives a total mass of roughly

$$
M_{\text {virial }}=6 \times 10^{7} M_{\odot}
$$

within a sphere of radius $0.8 \mathrm{pc}$.

This is, of course, just an order of magnitude estimate. Other estimates for the mass of the supermassive $\mathrm{BH}$ of $\mathrm{M} 31$ range from about $10^{6}$ to $10^{7} M_{\odot}$.

\subsection{Sagittarius $\mathbf{A}^{*}$}

Perhaps the most convincing evidence for a black hole comes from the center of our own galaxy that coincides with the enigmatic strong radiosource Sgr A*. The existence of a dark massive object at the center of the Galaxy has been inferred from the motions of stars and gas in its vicinity. The motions of the stars were observed and recorded for many years by two independent groups [63, 64, 65, 66, 67]. High-resolution infrared observations made it possible to follow the orbits of individual stars around this object (Schödel et al. [63, 64]; Ghez et al. [66, 67]). The movies (1st movie courtesy R. Genzel; 2nd movie courtesy A. Ghez [68]) show time-elapsed images of the Galactic Center region revealing the (eccentric) orbits of several stars. 
The projected positions of the star S2(S0-2) that was observed during the last decade [63, 64] suggest that $\mathrm{S} 2(\mathrm{~S} 0-2)$ is moving on a Keplerian orbit with a period $P$ of $15.2 \mathrm{yr}$ around $\mathrm{Sgr} \mathrm{A}^{*}$ and the estimated semimajor axis $a$ of the order of $4.62 \mathrm{mpc}$.

Then, neglecting the star mass, Kepler's third law (5.2) gives

$$
M_{\mathrm{SgrA} *}=\frac{4 \pi^{2} a^{3}}{G P^{2}}=3.7 \times 10^{6} M_{\odot} .
$$

The salient feature of the new adaptive optics data is that, between April and May 2002, S2(S02) apparently sped past the point of closest approach with a velocity $v \sim 6000 \mathrm{~km} / \mathrm{s}$ at a distance of about 17 light-hours [63] or $123 \mathrm{AU}$ from Sgr A*. This implies that an enormous mass of the central object is concentrated in a very small volume, strongly suggesting that the object is a $\mathrm{BH}$.

Another star, S0-16 (S14), which was observed during the last few years by Ghez et al. [67] with the Keck telescope in Hawaii, recently made a spectacular U-turn, crossing the point of closest approach at an even smaller distance of 8.32 light-hours or $60 \mathrm{AU}$ from Sgr A* with a velocity $v$ $\sim 9000 \mathrm{~km} / \mathrm{s}$. Ghez et al. thus conclude that the gravitational potential around Sgr A* has an approximately $r^{-1}$ form, for radii larger than $60 \mathrm{AU}$, corresponding to $1169 R_{\mathrm{SCH}}$, where $R_{\mathrm{SCH}}=$ $2 M=0.051 \mathrm{AU}$ for $M=2.6 \times 10^{6} M_{\odot}$.

\subsection{Supermassive BHs in Active Galactic Nuclei}

Many galaxies possess extremely luminous central regions, with luminosity (in particular the luminosity of X-ray radiation) exceeding the luminosity of ordinary galaxies by several orders of magnitude. These luminous central regions are called active galactic nuclei (AGN). To this class belong the so-called quasistellar objects (QSO) and quasars ${ }^{6}$.

What makes these galaxies "active" is the emission of enormous amounts of energy from their nuclei. Moreover, the luminosities of active galactic nuclei fluctuate on very short time scales within days or sometimes even minutes. The time variation sets an upper limit to the size of the emitting region. For this reason, we know that the emitting regions of active galactic nuclei are only light-minutes or light-days across, making them less than one ten-millionth the size of the galaxy in which they sit.

How could a luminosity hundreds of times that of an entire galaxy be emitted from a volume billions of times smaller? Of all proposed explanations, only one has survived close scrutiny: the release of gravitational energy by matter falling towards a black hole [49, 69]. Even using an energy source as efficient as gravity, the black holes in active galactic nuclei would need to be supermassive in order to produce the luminosities of quasars.

\subsubsection{Eddington Limit}

The most efficient way of generating energy is by the release of gravitational potential energy through mass accretion. For example, a simple calculation shows that for matter falling straight down onto the surface of a $1.4 M_{\odot}$ neutron star, about $21 \%$ of the rest mass is released. This is almost 30 times larger than the energy that hydrogen fusion can provide.

\footnotetext{
${ }^{6}$ Quasar is short for quasistellar radio source. Quasars are radio-loud whereas QSOs are radio-quiet . These names are sometimes confused in the literature. Both terms, $Q S O$ and quasar, are often used to refer to both types of objects $[6]$.
} 
The efficiency may be even larger if the matter is accreted through an accretion disk of a rotating black hole. The accretion luminosity (i.e., the radiation energy released per unit of time) generated by a mass accretion rate, $\dot{M}$, through the disk may be written as

$$
L_{\mathrm{disk}}=\eta \dot{M}
$$

where $\eta$ is the radiative efficiency of the disc equal to the binding energy of the innermost stable circular orbit per unit rest mass. As we have shown in section 4.1.3, $0.0572<\eta<0.423$, with the lower and upper bounds for a nonrotating and for a maximally rotating $\mathrm{BH}$, respectively.

However, the radiation is interacting with the accreting gas and there is a limit to the luminosity above which the radiation pressure, acting against gravitational attraction, exceeds gravity and thereby stops the accretion.

Consider a fully ionized hydrogen plasma accreting near the surface of a compact object of mass $M$. The upward force on the infalling matter is mainly due to the interaction of radiating photons with electrons in the plasma. If the photon luminosity is $L$, the number of photons $N_{\gamma}$ crossing unit area per unit time at radius $r$ is

$$
N_{\gamma}=\frac{L}{\varepsilon_{r} 4 \pi r^{2}},
$$

where $\varepsilon_{r}$ is the mean energy transferred radially per collision. The number of collisions per electron per unit time is $\sigma N_{\gamma}$, where $\sigma$ is the photon-electron scattering cross section. The force per electron is just the rate at which the momentum is deposited radially per unit time, so we multiply by $p_{r}=\varepsilon_{r}$ to obtain

$$
F=\sigma N_{\gamma} p_{r}=\frac{L \sigma}{4 \pi r^{2}} .
$$

In order for accretion to occur, the gravitational force per electron (acting via the proton)

$$
F_{\text {grav }}=-\frac{\mathscr{M} m_{p}}{r^{2}}
$$

must exceed the radiation force (6.12). Here, $\mathscr{M} \simeq M$ is the enclosed mass at radius $r$. Equating the radiation force (6.12) with the gravitational force (6.13) sets an upper bound to accretion luminosity known as the Eddington limit

$$
L_{\mathrm{Edd}}=4 \pi \frac{\mathscr{M} m_{p}}{\sigma} .
$$

The dominant electron-photon process in a highly ionized hydrogen gas is the scattering of photons by free electrons (Thomson scattering) with the cross section

$$
\sigma_{\mathrm{T}}=\frac{8 \pi}{3}\left(\frac{e^{2}}{m_{e}}\right)^{2}=6.65 \times 10^{-25} \mathrm{~cm}^{2} .
$$

This gives

$$
L_{\mathrm{Edd}}=1.3 \times 10^{38}\left(\frac{M}{M_{\odot}}\right) \mathrm{erg} \mathrm{s}^{-1}
$$




\section{Relativistic Eddington Limit}

In a strong gravitational field the expression (6.13) for the gravitational force is no longer valid. Here we derive the general relativistic expression for the force per electron (acting via the proton) under the assumption that the energy density of the fluid is still nonrelativistic, i.e., $\rho=m_{p} n$ and $p \ll \rho$.

Consider a spherical shell of radius $r$ with thickness $d r$. The force acting on the shell element of surface area $d S$ is

$$
d F=d S d p=d \mathscr{V}(1-2 \mathscr{M} / r)^{1 / 2} \frac{d p}{d r}
$$

where

$$
d \mathscr{V}=d r d S(1-2 \mathscr{M} / r)^{-1 / 2}
$$

is the proper volume element and $d p$ is the difference between radial pressures at $r$ and $r+d r$. Next, we substitute $d p / d r$ by the right-hand side of the TOV equation (2.18) in which we neglect the pressure (since $p \ll \rho$ by assumption). According to (2.11), the number of particles in the shell element is $d \mathscr{N}=n d \mathscr{V}$. Then, the gravitational force per particle is

$$
F_{\text {grav }}=\frac{d F}{d \mathscr{N}}=-\frac{m_{p} \mathscr{M}}{r^{2}}(1-2 \mathscr{M} / r)^{-1 / 2} .
$$

Equating $\left|F_{\text {grav }}\right|$ thus obtained with the photon force (6.12), we find

$$
L_{\mathrm{Edd}}=\frac{4 \pi m_{p} \mathscr{M}}{\sigma}(1-2 \mathscr{M} / r)^{-1 / 2}
$$

Hence, compared with (6.14), the Eddington limit in the strong gravitational field is larger by a relativistic correction factor.

\subsubsection{Radius and Mass Estimate}

The luminosity of a typical quasar varies in time with a typical period of $1 \mathrm{hr}$. This sets the upper limit to the radius of about

$$
R \simeq 7 \mathrm{AU}=1.1 \times 10^{9} \mathrm{~km} .
$$

Considering that AGNs are the most luminous objects known, this is an incredibly small size.

The typical quasar luminosity of $5 \times 10^{46} \mathrm{erg} \mathrm{s}^{-1}$ is equivalent to more than 500 galaxies of the Milky Way size! Now, the constraint that the luminosity must be less than the Eddington limit, $L<L_{\text {Edd }}$, provides a lower limit for the mass of the central object

$$
M>M_{0}=3.3 \times 10^{8} M_{\odot} .
$$

The mass limit $M_{0}$ is quite close to the mass $M_{\mathrm{BH}}=R /(2 G)=3.7 \times 10^{8} M_{\odot}$ of a BH the Schwarzschild radius of which is equal to the radius of the quasar $R$ estimated above. This fact supports the idea that supermassive BHs are responsible for powering AGNs. (Carroll and Ostlie [6], p. 1181)

The estimated value of the lower mass limit is somewhat smaller if the general relativistic correction to the Eddington limit is taken into account. Using the relativistic expression (6.20), we find

$$
M>M_{0}\left(\sqrt{1+\frac{M_{0}^{2}}{4 M_{\mathrm{BH}}^{2}}}-\frac{M_{0}}{2 M_{\mathrm{BH}}}\right)=2.14 \times 10^{8} M_{\odot} .
$$




\subsubsection{Estimate of the AGN Efficiency}

According to equation (6.10), the radiative efficiency $\eta$ of an accretion disk is defined as the energy it radiates per unit accreted mass. As shown in section 4.1.3, $\eta$ equals the binding energy of gas at the ISCO, which in turn depends on the $\mathrm{BH}$ spin parameter $a$.

In a typical accretion system, one can easily measure the accretion luminosity $L_{\mathrm{disc}}$ (provided the distance is known), but one practically never has an accurate estimate of the mass accretion rate $\dot{M}$, so one cannot calculate $\eta$ for an individual AGN with the precision needed to estimate $a$. However, a rough estimate can be made for an average AGN. From observations of high redshift AGN, one can estimate the mean energy radiated by supermassive BHs per unit volume of the universe. Similarly, by taking a sample of supermassive BHs in nearby galaxies, one can estimate the mean mass in BHs per unit volume of the universe at present. Assuming that supermassive BHs acquire most of their mass via accretion (a not unreasonable hypothesis), one can divide the two quantities to obtain the mean radiative efficiency of AGN. The current data suggest an efficiency $\eta \sim 0.1-0.15$ for supermassive BHs on average (Elvis et al [70], Yu and Tremaine [71]). Such large values of $\eta$ are possible only if supermassive BHs have significant rotation.

It should be noted that this is only a statistical result for the population of AGN as a whole, and the method does not say anything about the rotation of any specific $\mathrm{BH}$.

\section{Intermediate Mass BHs}

So far we have been concerned with either the stellar mass BHs $\left(M \sim 3.5-20 M_{\odot}\right)$ or the supermassive BHs $\left(M>10^{6} M_{\odot}\right)$. Are there BHs in the intermediate mass range $10^{2}-10^{4} M_{\odot}$ ? There is a priori no reason for such BHs not to exist.

There is a tentative evidence based on the Eddington limit that the intermediate mass BHs do exist. In several nearby galaxies a number of ultraluminous X-ray sources have been detected [72]. As their luminosities of the order of $10^{40}$ or more exceed the Eddington limit (6.16) of $10 \mathrm{M}_{\odot}$ $\mathrm{BHs}$, these objects are argued to be good candidates for the intermediate mass BHs [73]. However, large apparent luminosity may, under special conditions, be produced by stellar mass BHs, e.g, by a supercritical accretion (for details and references, see Poutanen et al. [74]). Hence, it is at present unclear what exactly the ultraluminous X-ray sources are. Dynamical mass measurements would settle the issue but, unfortunately, none of the sources has a binary companion to provide a robust mass estimate.

\section{Observational Evidence for the Horizon}

To prove that a $\mathrm{BH}$-like object is indeed a $\mathrm{BH}$, one needs to demonstrate that it possesses an event horizon. The major tests for a $\mathrm{BH}$ horizon are based on i) advection-dominated accretion flows (ADAF), ii) X-ray bursts, and iii) direct imaging $[8,75,76]$

\subsection{ADAF}

Advection dominated accretion flows $[77,78]$ describe accretion with very low radiative efficiency in which the energy released by viscosity friction removing the angular momentum from 
the accreting matter is not radiated away but stored in the flow. If an ADAF forms around a BH, the stored energy will be lost forever under the event horizon, whereas if the accreting object is a NS, this energy must be radiated away once matter lands on its surface. Therefore, the argument goes, BHs should be dimmer than NSs if an ADAF is present in both cases.

Narayan, Garcia, and McClintock [79] suggested that precisely such a comparison could be done using X-ray binaries. Most of the known stellar-mass BH candidates are in a class of X-ray binaries called $\mathrm{X}$-ray novae. These systems are characterized by a variable mass accretion rate, and tend to spend most of their time in a quiescent state with a very low accretion rate $\dot{M}$ and accretion luminosity $L_{\text {acc }}$. Only occasionally do they go into outburst, when they accrete with high $\dot{M}$ and become bright. Spectral observations of quiescent BH binaries can be explained in terms of an ADAF. Narayan et al. [79] compared quiescent luminosities of X-ray binaries supposed to contain BHs with those of neutron-star X-ray binaries and realized that, in accordance with the prediction of the ADAF model, systems containing black-hole "candidates" are dimmer. They came to the conclusion that they found evidence for the presence of event horizons. However, this conclusion has been challenged (for details and references, see $[8,75]$ ).

\subsection{X-ray Bursts}

In some X-ray binaries, X-ray bursts are observed in addition to the quiescent X-ray luminosity. In a typical X-ray burst luminosity increases up to nearly the Eddington limit in less than a second, and the flux then declines over a period of a few seconds or a few tens of seconds. Remarkably, no X-ray bursts of this kind were observed in any BH binary. Narayan and Heyl [80] argued that the lack of bursts is a strong evidence for the horizon in $\mathrm{BH}$ candidates.

The explanation for such an absence of X-ray bursts is quite simple. At present, it is widely accepted that X-ray bursts arise from thermonuclear detonation of accreted material [80, 81]. Accreted material builds up on the surface of the compact object and is compressed by the object's gravity. After sufficient material accumulates, it undergoes unstable thermonuclear burning, which we observe as an X-ray burst.

A key point is that the compact object must have a surface. Material cannot accumulate on an event horizon, and so no bursts can come from an X-ray binary whose compact object is a BH. For more details, references, and critical discussion, see $[8,75]$

\subsection{Direct Imaging}

The most promising line of search for a direct evidence is to construct an image of the region near the event horizon using interferometry.

For definiteness, consider a nonrotating $\mathrm{BH}$ with a horizon at radius $R$. Because of strong gravitational lensing in the vicinity of the $\mathrm{BH}$, a distant observer will see an apparent boundary of the $\mathrm{BH}$ at a radius of $3 \sqrt{3} R / 2$ [82]. Rays with impact parameters inside this boundary intersect the horizon, while rays outside the boundary miss the horizon. The angular size of the boundary, e.g., for $\mathrm{Sgr} \mathrm{A}^{*}$ with $M \sim 4 \times 10^{6} M_{\odot}$ and at a distance of $8 \mathrm{kpc}$, is $\sim 0.02$ mas which is not beyond reach. The supermassive $\mathrm{BH}$ in the nucleus of the nearby $(15 \mathrm{Mpc})$ giant elliptical galaxy M87, with a mass of $3 \times 10^{9} M_{\odot}$ and an expected angular size of $\sim 0.01$ mas, is another object of interest [83]. 
The best angular resolution achievable today is with radio interferometry, where angles less than 1 mas are routinely resolved. In the not too distant future, it should be possible to operate interferometers at wavelengths $\lambda<1 \mathrm{~mm}$ and with baselines as large as the diameter of the Earth $b \sim 10^{4} \mathrm{~km}$. For details, see Falcke et al. [82].

\section{Alternatives to Supermassive BHs}

Given the accumulated evidence for supermassive compact objects ranging from a few $10^{6} M_{\odot}$ to a few $10^{9} M_{\odot}$, the existence of black-hole-like objects is beyond doubt $[53,56]$. What still remains an issue is whether these supermassive objects are BHs with the Schwarzschild (or Kerr) metric describing the physics of the interior, or some other objects built out of more or less exotic substance but with a regular behavior in the interior. A standard astrophysical scenario in the form of a compact cluster of dark stars (e.g., neutron stars or brown dwarfs) although not entirely excluded, is quite unlikely. It has been demonstrated that, in the case of NGC 4258 and our Galaxy, such a cluster would be short-lived and would either "evaporate" or become a BH in much less time than the age of the Galaxy [84].

A number of alternatives to classical BHs have been proposed with no singularities in the interior. The three representative models are: 1) Neutrino (or neutralino) stars, 2) Boson stars, 3) Dark energy stars

\subsection{Neutrino Stars}

Here we use the term neutrino stars as a generic name for any degenerate fermion star composed of neutral weakly interacting fermions, e.g., neutrinos or supersymmetric fermionic partners such as neutralinos, gravitinos, and axinos. The simplest model proposed for supermassive compact objects at the galactic centers is a self-gravitating degenerate fermion gas composed of, e.g., heavy sterile neutrinos $[85,86,87,88,89]$. Sterile neutrinos in the keV mass range have recently been extensively discussed as dark-matter candidates $[90,91]$

As we have seen in the example of a neutron star, a self-gravitating ball of degenerate fermionic matter is supported against gravitational collapse by the degeneracy pressure of fermions due to the Pauli exclusion principle, provided the total mass is below the OV limit (3.14), with an $m^{-2}$ functional dependence on the fermion mass $m$.

Let us assume that the most massive objects are sterile neutrino stars at the OV limit with $M_{\mathrm{OV}} \simeq 3 \times 10^{9} M_{\odot}$, such as the supermassive compact dark object at the center of the radio-galaxy M87 [57]. Using (3.14) we find that the neutrino mass required for this scenario is

$$
\begin{array}{ll}
m \simeq 14 \mathrm{keV} & \text { for } g=2, \\
m \simeq 12 \mathrm{keV} & \text { for } g=4
\end{array}
$$

From (3.14) and (3.15) it follows that a neutrino star of mass $M_{\mathrm{OV}}=3 \times 10^{9} M_{\odot}$ would have a radius $R_{\mathrm{OV}}=4.4466 R_{\mathrm{Sch}}=3.9396 \times 10^{10} \mathrm{~km}=1.52$ light-days, where $R_{\mathrm{Sch}}=2 G M_{\mathrm{OV}}$ is the Schwarzschild radius for $M_{\mathrm{OV}}$. Thus, at a distance of a few Schwarzschild radii away from the supermassive object, there is little difference between a neutrino star at the OV limit and a BH, in particular since the last stable orbit around a $\mathrm{BH}$ already has a radius of $3 R_{\mathrm{Sch}}$. 
Now, we wish to investigate the possibility that this scenario can be extrapolated to explain all observed supermassive $\mathrm{BH}$, and in particular $\mathrm{Sgr} \mathrm{A}^{*}$, the supermassive compact dark object at the Galactic center. As the mass of $\mathrm{Sgr} \mathrm{A}^{*}$ is by about a factor of $10^{3}$ lower than the OV limit above, we can use the Newtonian mass-radius scaling relation (2.32) derived from the Lane-Emden equation (2.28) with the polytropic index $n=3 / 2$. We find

$$
R=4.51 \frac{m_{\mathrm{Pl}}^{2}}{m^{8 / 3} M^{1 / 3}}\left(\frac{2}{g}\right)^{2 / 3}=7.1406 \times 10^{5}\left(\frac{12 \mathrm{keV}}{m}\right)^{8 / 3}\left(\frac{4}{g}\right)^{2 / 3}\left(\frac{M_{\odot}}{M}\right)^{1 / 3} \mathrm{AU} .
$$

Here, $1 \mathrm{mpc}=206.265 \mathrm{AU}$. The degeneracy factor $g=2$ describes either spin $1 / 2$ fermions (without antifermions) or spin 1/2 Majorana fermions. For Dirac fermions (or spin 3/2 Majorana fermions), we have $g=4$. Using the canonical value $M=2.6 \times 10^{6} M_{\odot}$, we find $R \simeq 5200 A U$ to be compared with the observational upper limit [67] $R \leq 60 \mathrm{AU}$ for Sgr A*. Hence, a neutrino star made of $m=12-15 \mathrm{keV}$ neutrinos is ruled out as an explanation for Sgr $\mathrm{A}^{*}$.

To be able to explain Sgr A* as a neutrino star, we need a minimal fermion mass $m_{\min }=63.9$ $\mathrm{keV} / \mathrm{c}^{2}$ for $g=4$. The maximal mass of a neutrino star made of these fermions, as given by the $\mathrm{OV}$ limit (3.14), is [92]

$$
M_{\mathrm{OV}}^{\max }=1.083 \times 10^{8} M_{\odot} .
$$

Clearly, a neutrino star scenario which would cover the whole mass-radius range of compact supermassive galactic centers is ruled out. However, a "hybrid" scenario [92] in which all supermassive compact dark objects with masses $M>M_{\mathrm{OV}}^{\max }$ are black holes, while those with $M \leq M_{\mathrm{OV}}^{\max }$ are neutrino stars, is not excluded.

At first sight, such a hybrid scenario does not seem to be particularly appealing. However, it is important to note that a similar scenario is actually realized in nature, with the co-existence of neutron stars with masses $M \lesssim 3 M_{\odot}$, and stellar-mass BHs with mass $M \gtrsim 3 M_{\odot}$, as observed in binary systems in the Galaxy.

An indirect support for this scenario is the absence of clear evidence for intermediate mass $\mathrm{BH}$ candidates, which is difficult to explain in the conventional $\mathrm{BH}$ scenario (in which BHs are all baryonic). If the hybrid scenario were realized in nature, the intermediate mass neutrino stars would exist but having very large radii would be rather dilute and hence difficult to detect.

\subsection{Boson Stars}

Boson stars are static configurations of self-gravitating (with or without self-interaction) complex scalar fields. In self-interacting scalar field theories, such as $\phi^{4}$ theory, there are cases where a homogeneous condensate is a stable ground state, known as the Bose-Einstein (BE) condensate. Hence, a boson star being basically a self-gravitating BE condensate may be regarded as a $T \rightarrow 0$ limit of a self-gravitating boson gas [93]. The ground state of a condensed cloud of charged bosons of mass $m$, interacting only gravitationally and having a total mass $M$ below a certain limit of the order $M_{\mathrm{Pl}}^{2} / m$, is a stable spherically symmetric configuration [94] which is usually referred to as mini-soliton star [95] or mini-boson star [96]. For a recent review and a comprehensive list of references, see Schunck and Mielke [97].

The gravitational collapse of a self-interacting BE condensate is prevented by a repulsive selfinteraction, e.g., in the form of $\lambda|\Phi|^{4}$. That makes it astrophysically interesting as its maximal mass is $\sim m_{\mathrm{Pl}}^{3} / m_{B}^{2}$, hence comparable with the mass of a neutron star or a neutrino star. 
Seidel and Suen have demonstrated that boson stars may be formed through a dissipationless mechanism, called gravitational cooling [98]. Boson stars have recently attracted some attention as they may well be candidates for nonbaryonic dark matter [99].

The action of the gravitationally coupled complex scalar field $\Phi$ reads

$$
S_{\mathrm{BS}}=\int d^{4} x \sqrt{-\operatorname{det} g}\left(-\frac{R}{16 \pi}+\mathscr{L}_{\mathrm{KG}}\right),
$$

with the Klein-Gordon Lagrangian

$$
\mathscr{L}_{\mathrm{KG}}=g^{\mu v}\left(\partial_{\mu} \Phi^{*}\right)\left(\partial_{\nu} \Phi\right)-m^{2}|\Phi|^{2}-U\left(|\Phi|^{2}\right) .
$$

By varying the action with respect to $\Phi$ one obtains the Klein-Gordon equation

$$
\left(\square+\frac{d U}{d|\Phi|^{2}}\right) \Phi=0
$$

and the variation with respect to $g_{\mu \nu}$ yields Einstein's equations (A.24) in which

$$
T_{\mu \nu}=\left(\partial_{\mu} \Phi^{*}\right)\left(\partial_{\nu} \Phi\right)+\left(\partial_{\mu} \Phi\right)\left(\partial_{\nu} \Phi^{*}\right)-g_{\mu \nu} \mathscr{L}_{\mathrm{KG}},
$$

Although this energy-momentum tensor is not in the perfect fluid form (B.1, we can still identify the radial pressure $p$ and the density $\rho$ with $-T_{r}^{r}$ and $T_{0}^{0}$, respectively.

Given the interaction potential $U$, one has to solve a coupled system of Klein-Gordon and Einstein equations in spherically symmetric static spacetime. Unlike in the case of a fermion star, where the information about matter was provided by the equation of state, the matter here is described by the KG equation (9.6). The stationarity ansatz

$$
\Phi(r, t)=\frac{1}{\sqrt{2}} \varphi(r) e^{-i \omega t}
$$

describes a spherically symmetric bound state of the scalar field, where the frequency $\omega$ is determined by the asymptotic condition $\varphi(r) \rightarrow 0$ as $r \rightarrow \infty$.

It turns out that even the simplest case $U=0$ has a nontrivial ground-state solution, called a mini-boson star. The gravitational collapse of a mini-boson star is prevented by Heisenberg's uncertainty principle. This provides us also with crude mass estimates: For a boson to be confined within the star of radius $R_{0}$, the Compton wavelength has to satisfy $\lambda_{\Phi}=2 \pi / m \simeq 2 R_{0}$. On the other hand, the star's radius should be larger than the Schwarzschild radius, $R_{0}>R_{\mathrm{Sch}}=2 M / m_{\mathrm{Pl}}^{2}$ in order to avoid instability against complete gravitational collapse. In this way we obtain an estimate

$$
M<\frac{\pi}{2} m_{\mathrm{Pl}}^{2} / m .
$$

This upper bound is slightly larger than the so-called Kaup limit,

$$
M_{\max }=0.633 m_{\mathrm{Pl}}^{2} / m=8.4639 \times 10^{-10}\left(\frac{1 \mathrm{eV}}{m}\right) M_{\odot}
$$

obtained numerically [96]. Clearly, mini-boson stars are irrelevant to astrophysical context, unless the boson mass $m$ is ridiculously small. 
This result was later extended by Colpi et al. [100] for interacting bosons with a quartic selfinteraction

$$
U=\lambda|\Phi|^{4}
$$

In this case, the maximal mass is obtained as $[100,101]$

$$
M_{\max }=\frac{1}{\sqrt{8 \pi^{3}}} \sqrt{\lambda} \frac{m_{\mathrm{Pl}}^{3}}{m^{2}}=0.1 \sqrt{\lambda}\left(\frac{1 \mathrm{GeV}}{m}\right)^{2} M_{\odot}
$$

quite similar to the fermion star mass limit. The effective radius of an interacting boson star in the Newtonian regime turns out to be independent of central density and in a good approximation is equal to

$$
R_{\text {eff }}=\frac{\pi}{\sqrt{8 \pi}} \sqrt{\lambda} \frac{m_{\mathrm{Pl}}}{m^{2}}=1.5096 \sqrt{\lambda}\left(\frac{1 \mathrm{GeV}}{m}\right)^{2} \mathrm{~km} .
$$

Hence, boson stars with a quartic self-interaction all have the same radius. This behavior is quite different from the fermion star case, where the radius scales with the star mass as $R \sim M^{1 / 3}$ (see equation (9.2)).

The results are summarized in the table

\begin{tabular}{|l|c|c|}
\hline & Maximum & Effective \\
Object & Mass & Radius \\
\hline Fermion Star & $M_{\mathrm{OV}}=0.384 m_{\mathrm{PL}}^{3} / m^{2}$ & $R_{\mathrm{OV}}=3.36 m_{\mathrm{Pl}} / m^{2}$ \\
Mini-Boson Star & $M_{\mathrm{Kaup}}=0.633 m_{\mathrm{PL}}^{2} / m$ & $R \sim 1 / m$ \\
Boson Star & $M_{\max }=1 /\left(\sqrt{8 \pi^{3}}\right) \sqrt{\lambda} m_{\mathrm{Pl}}^{3} / m^{2}$ & $R_{\mathrm{eff}}=\pi /(\sqrt{8 \pi}) \sqrt{\lambda} m_{\mathrm{Pl}} / m^{2}$ \\
\hline
\end{tabular}

Could boson stars fit the whole range of compact supermassive galactic centers? To answer this question, we proceed as in the case of neutrino stars. We assume that the most massive objects are boson stars with maximal mass, i.e., $M_{\max } \simeq 3 \times 10^{9} M_{\odot}$, for the supermassive compact dark object at the center M87. From this we find

$$
\sqrt{\lambda}\left(\frac{1 \mathrm{GeV}}{m}\right)^{2}=3 \times 10^{10}
$$

which also fixes the radius given by (9.13)

$$
R=4.53 \times 10^{10} \mathrm{~km}=303 \mathrm{AU}
$$

quite close to the radius $R_{\mathrm{OV}}$ of the neutrino star discussed in section 9.1. Since the radius of a boson star does not depend on its mass, this value should also fit the radii of all supermassive BHs. However, it obviously does not fit the observed radius limit of $\mathrm{Sgr} \mathrm{A}^{*}, R<60 \mathrm{AU}$, and even less it fits the radius of a typical AGN of $7 \mathrm{AU}$ (section 6.3.2).

Hence, boson stars with a quartic self-interaction are ruled out as a unique explanation for compact supermassive objects at galactic centers and AGN. Again, it is not excluded that some of the galactic centers harbor a boson star. 


\subsection{Dark-Energy Stars}

Dark energy (DE) is a substance of negative pressure needed for an accelerated cosmology. In order to achieve acceleration, DE must satisfy an equation of state $p_{\mathrm{de}}=w \rho_{\mathrm{de}}$, where $w$ may depend on the cosmological scale $a$ but at present $(a=1) w<-1 / 3$. In addition, one also expects that DE satisfies the dominant energy condition, in which case the equation of state satisfies $w \geq-1$. Nevertheless, matter with $w<-1$ dubbed "phantom energy" [102, 103] has recently received attention as it might fit the most recent SN 1a data slightly better than the usual DE.

In cosmological context, $\rho_{\mathrm{de}}$ is homogeneous and it is normally assumed that DE does not cluster. However, it is not excluded that owing to gravitational instability, small inhomogeneities (analogous to dark-matter inhomogeneities) grow and build structure, e.g., in the form of spherical configurations. Hence, we define dark-energy stars as spherical solutions to Einstein's equation with matter described by the DE equation of state [104, 105]. It is worth noting that DE could be described by a scalar field theory (quintessence) with a suitably chosen interaction potential and/or with a noncanonical kinetic energy term in the Lagrangian. This scalar field theory would correspond to an effective equation of state with desirable properties [106]. In this way, DE stars could be regarded as boson stars but with a rather unusual self-interaction.

Here, we briefly discuss two examples of DE stars: de Sitter gravastars and Chaplygin gravastars.

\subsubsection{De Sitter Gravastars}

The simplest example of DE star is a gravitating vacuum star or a gravastar. Chapline et al. $[107,108]$ put forth an interesting proposal based on analogies to condensed matter systems where the effective general relativity was an emergent phenomenon. Specifically, assuming the Schwarzschild exterior, they suggested that the sphere where the lapse function $\xi=g_{00}^{1 / 2}$ vanished marked a quantum phase transition, $\xi$ increasing again at $r<2 G M$. As this required negative pressure, the authors of [108] assumed the interior vacuum condensate to be described by de Sitter space with the equation of state $p=-\rho$.

Subsequently, the idea of gravitational vacuum condensate, or 'gravastar', was taken up by Mazur and Mottola [109, 110] and Dymnikova [111], replacing the horizon with a shell of stiff matter astride the surface at $r=2 G M$. Visser and Wiltshire [112] and recently Carter [113] also examined the stability of the gravastar using the Israel thin-shell formalism [114].

Given the mass M, the interior is described by a solution, similar to the the Einstein-de Sitter universe, with constant density $\rho=\rho_{0}$ up to the de Sitter radius $R_{\mathrm{dS}}=2 M$ (actually slightly further out). Hence, the interior is de Sitter, $\rho_{0}$ being the vacuum energy density. The lapse function is given by

$$
\xi=\left(1-\frac{r^{2}}{R_{\mathrm{dS}}^{2}}\right)^{1 / 2},
$$

with

$$
R_{\mathrm{dS}}=\sqrt{\frac{3}{8 \pi}} \rho_{0}^{-1 / 2}
$$

In order to join the interior solutions to a Schwarzschild exterior at a spherical boundary of radius $R$, it is necessary to put a thin spherical shell at the boundary with a surface density and a 
surface tension satisfying Israel's junction conditions [114]. As the pressure does not vanish at the boundary, it must be compensated by a negative surface tension of the shell.

The gravastar has no horizon or singularity! Its surface is located at a radius just slightly larger than the Schwarzschild radius $R=2 M+\varepsilon, \varepsilon \sim 2 l_{\mathrm{Pl}}$. Hence, there is practically no observational way to distinguish a Schwarzschild BH from a gravastar. Basically, any BH-like object observed in nature may be a gravastar.

In spite of these attractive features the gravastar has been met with a cool reception. Certainly, the assumption of a de Sitter interior presents a quandary: on the one hand, the quantum phase transition would suggest that the associated cosmological constant is a fundamental parameter; on the other hand, to accommodate the mass range of supermassive black-hole candidates, it must vary over some six orders of magnitude. In addition, the notorious cosmological constant problem is reversed for gravastars: why is the vacuum energy in the interior of a gravastar so much larger than the observed vacuum energy density in the universe? If we identify the most massive black-hole candidate observed at the center of M87, with mass $M_{\max }=3 \times 10^{9} M_{\odot}$, with the de Sitter gravastar, then $\rho_{0}=(9.7 \mathrm{keV})^{4}$, to be contrasted with the $\left(10^{-3} \mathrm{eV}\right)^{4}$ values wanted for cosmology. Another question is how does a gravastar form? The entropy of a stellar mass gravastar is much less than the entropy of an ordinary star and this would require an extremely efficient cooling mechanism before gravastars could form during stellar collapse [75].

\subsubsection{Chaplygin Gravastars}

Consider a particular form of DE with a rather peculiar equation of state

$$
p=-\frac{A}{\rho}
$$

Equation (9.18) describes the Chaplygin gas which, for $\rho \geq \sqrt{A}$, has attracted some attention as a dark-energy candidate [106, 115]. Consider a self-gravitating Chaplygin gas and look for static solutions. In particular, we look for static Chaplygin gas configurations in the phantom $(w<-1)$ regime, i.e., when

$$
\rho<\sqrt{A} .
$$

We show that these configurations could provide an alternative scenario for compact massive objects at galactic centers [116]. Moreover, equation (9.18) yields the de Sitter gravastar solution in the limit when the central density of the static solution approaches the value $\sqrt{A}$.

Combining (9.18) with the TOV equations (2.18), and (2.19), one has

$$
\begin{gathered}
\rho^{\prime}=\left(1-\frac{\rho^{2}}{A}\right)\left(\frac{\rho \mathscr{M}-4 \pi A r^{3}}{r(r-2 \mathscr{M})}\right), \\
\frac{d \mathscr{M}}{d r}=4 \pi r^{2} \rho .
\end{gathered}
$$

In Fig. 4 we exhibit the resulting $\rho(r)$ for selected values of $\rho_{0} / \sqrt{A}$. The solutions depend essentially on the magnitude of $\rho_{0}$ relative to $\sqrt{A}$. In the following we summarize the properties of three classes of solutions corresponding to whether $\rho_{0}$ is larger, smaller, or equal to $\sqrt{A}$. 


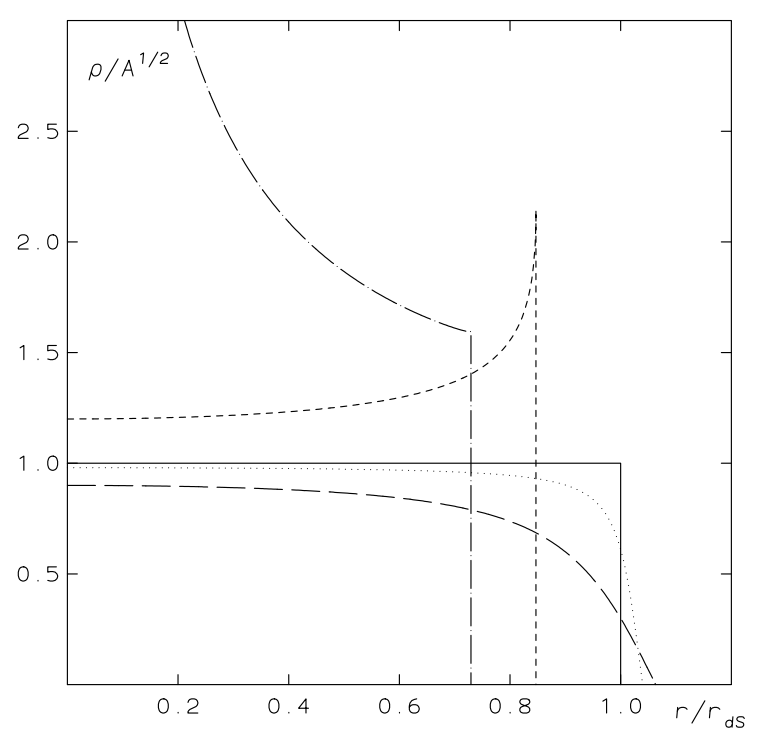

Figure 4: Density profile of the Chaplygin gravastar for $\rho_{0} / \sqrt{A}=1.2$ (short dashed), 0.98 (dotted), 0.9 (long dashed). The limiting singular solution with the $r^{-2 / 3}$ behavior at small $r$ is represented by the dot-dashed and the de Sitter gravastar by the solid line.

i) For $\rho_{0}>\sqrt{A}$, the density $\rho$ increases and the lapse function $\xi$ decreases with $r$ starting from the origin up to the black-hole horizon radius $R_{\mathrm{bh}}$, where $2 G \mathscr{M}\left(R_{\mathrm{bh}}\right)=R_{\mathrm{bh}}$. In the limit $\rho_{0} \rightarrow \infty$, a limiting solution exists with a singular behavior

$$
\rho(r) \simeq\left(\frac{7 A}{18 \pi G r^{2}}\right)^{1 / 3}
$$

near the origin.

ii) For $\rho_{0}<\sqrt{A}$, both $\rho$ and $\xi$ decrease with $r$ up to the radius $R_{0}$ where they vanish. At that point the pressure $p$ blows up to $-\infty$ owing to (9.18). The enclosed mass $\mathscr{M}$ is always less than $r /(2)$, never reaching the black-hole horizon, i.e., the radius where $2 \mathscr{M}(r)=r$.

iii) For $\rho_{0}=\sqrt{A}$, the density $\rho$ remains constant equal to $\sqrt{A}$ up to the de Sitter radius $R_{\mathrm{dS}}=$ $2 M$. Hence, we recover the de Sitter solution precisely as in the gravastar case.

As $\rho_{0} \rightarrow \sqrt{A}$ from above or from below, solutions i) or ii), respectively, converge to iii) except at the endpoint. The lapse function in iii) joins the Schwarzschild solution outside continuously, whereas in i) and ii) it happens discontinuously.

As in the case of a de Sitter gravastar, in order to join our interior solutions to a Schwarzschild exterior at a spherical boundary of radius $R$, it is necessary to put a thin spherical shell ${ }^{7}$ at the boundary with a surface density and a surface tension satisfying Israel's junction conditions [114].

Case ii), together with iii), is of particular interest as we would like to interpret the supermassive compact dark objects at the galactic centers in terms of phantom energy rather than in terms of a classical black hole. It is natural to assume that the most massive such object is described by

\footnotetext{
${ }^{7}$ It has recently been demonstrated that the joining can be made continuous without the presence of a thin shell for a gravastar made of the fluid with an anisotropic pressure [117, 118].
} 
the de Sitter gravastar, i.e., solution iii) (depicted by the solid line in Fig. 4). The radius of this object is $R_{\mathrm{dS}}$ equal to the Schwarzschild radius $2 M_{\max }$. Clearly, solutions belonging to class ii), can fit all masses $M<M_{\max }$. However, for the phenomenology of supermassive galactic centers it is important to find, at least approximately, the mass-radius relationship for these solutions. This may be done in the low central density approximation, i.e., $\rho_{0} \ll \sqrt{A}$, which is similar to the Newtonian approximation but, in contrast to the Newtonian approximation, one cannot neglect the pressure term in (2.18). Moreover, as may be easily shown, in this approximation $\mathscr{M} \ll r^{3} p$, so that the pressure term becomes dominant. Next, neglecting $2 \mathscr{M}$ with respect to $r$, as in the usual Newtonian approximation, equation (9.20) simplifies to $\rho^{\prime}=4 \pi A r$, with the solution

$$
\rho=\rho_{0}\left(1-\frac{r^{2}}{R_{0}^{2}}\right) ; \quad R_{0}^{2}=\frac{\rho_{0}}{2 \pi A},
$$

which gives a mass-radius relation

$$
\frac{M}{R_{0}^{5}}=\frac{16 \pi^{2}}{15} A=\text { constant }
$$

The mass-radius relationship $M \propto R_{0}^{5}$ which phantom gravastars obey, offers the prospect of unifying the description of all supermassive compact dark objects at the galactic centers, as Chaplygingas phantom gravastars with masses ranging from $M_{\min }=10^{6} M_{\odot}$ to $M_{\max }=3 \times 10^{9} M_{\odot}$. Indeed, assuming that the most massive compact dark object, observed at the center of M87, is a Chaplygin phantom gravastar near the de Sitter gravastar limit, with $R_{\max }=2 M_{\max }=8.86 \times 10^{9} \mathrm{~km}=8.21 \mathrm{lhr}$, the compact dark object at the center of our Galaxy, with mass $M_{\mathrm{GC}}=3 \times 10^{6} M_{\odot}$, would have a radius $R_{\mathrm{GC}}=2.06 \mathrm{lhr}$ if the scaling law (9.24) holds. This radius is well below the distances of the closest approach to Sgr A* which the stars SO-2 $\left(R_{\min }=17 \mathrm{lhr}=123 \mathrm{AU},[63,64]\right)$ and SO-16 $\left(R_{\min }=8.32 \mathrm{lhr}=60 \mathrm{AU}[66,67]\right)$ recently had and beyond which the Keplerian nature of the gravitational potential of $\mathrm{Sgr} \mathrm{A}^{*}$ is well established.

\section{A. Basic General Relativity}

\section{A.1 Geometry}

The geometry of spacetime is described by the metric

$$
d s^{2}=g_{\mu v} d x^{\mu} d x^{v}
$$

where $g_{\mu \nu}$ is the metric tensor. The spacetime curvature is defined through the Riemann curvature tensor

$$
R_{\tau \sigma \beta \gamma}=g_{\tau \alpha} R_{\sigma \beta \gamma}^{\alpha}
$$

defined by

$$
R_{\delta \beta \gamma}^{\alpha}=\partial_{\gamma} \Gamma_{\beta \delta}^{\alpha}-\partial_{\beta} \Gamma_{\delta \gamma}^{\alpha}+\Gamma_{\rho \gamma}^{\alpha} \Gamma_{\beta \delta}^{\rho}-\Gamma_{\rho \beta}^{\alpha} \Gamma_{\gamma \delta}^{\rho},
$$

where

$$
\Gamma_{\mu \nu}^{\sigma}=\frac{1}{2} g^{\lambda \sigma}\left[\partial_{\mu} g_{v \lambda}+\partial_{\nu} g_{\mu \lambda}-\partial_{\lambda} g_{\mu v}\right]
$$


are the Christoffel symbols. A contraction of two indices gives the Ricci tensor

$$
R_{\alpha \beta}=g^{\lambda v} R_{\lambda \alpha v \beta}
$$

and a further contraction of the Ricci tensor gives the scalar curvature

$$
\mathscr{R}=g^{\alpha \beta} R_{\alpha \beta} .
$$

\section{A.2 Covariant Derivative}

A covariant derivative $\nabla_{\mu}$ of a field $Y$ (in general, $Y$ may be a tensor of any rank) is defined by

$$
D Y=\left(\nabla_{\mu} Y\right) d x^{\mu} \equiv Y_{; \mu} d x^{\mu},
$$

where $D Y$ is an infinitesimal difference between the value of the field $Y$ at the point $x^{\mu}+d x^{\mu}$ and the quantity $Y\left(x^{\mu}\right)$ parallelly displaced from $x^{\mu}$ to $x^{\mu}+d x^{\mu}$. Hence, $D Y$ consists of two parts: one is the change of $Y$ due to the parallel displacement and the other is the difference $d Y=Y\left(x^{\mu}+\right.$ $\left.d x^{\mu}\right)-Y\left(x^{\mu}\right)$ due to the functional dependence on $x^{\mu}$. The latter part is basically related to the ordinary partial differentiation. The difference due to the parallel displacement is related to the curvature of spacetime and depends on the tensor nature of $Y$. For a scalar, vector, and second-rank tensor one finds: [119]

- $\operatorname{scalar} \varphi$

$$
\varphi_{; \mu}=\varphi_{, \mu}
$$

- vector $V_{\mu}$

$$
V_{\mu ; \nu}=V_{\mu, v}-\Gamma_{\mu \nu}^{\rho} V_{\rho}, \quad V_{; v}^{\mu}=V_{, v}^{\mu}+\Gamma_{\rho v}^{\mu} V^{\rho}
$$

- tensor $A_{\mu v}$

$$
A_{\mu v ; \rho}=A_{\mu v, \rho}-\Gamma_{\mu \rho}^{\sigma} A_{\sigma v}-\Gamma_{v \rho}^{\sigma} A_{\mu \sigma}, \quad A_{; \rho}^{\mu v}=A_{, \rho}^{\mu v}+\Gamma_{\sigma \rho}^{\mu} A^{\sigma v}+\Gamma_{\sigma \rho}^{v} A^{\mu \sigma} .
$$

The covariant d'Alembertian is given by

$$
\square \equiv g^{\mu v} \nabla_{\mu} \nabla_{v}=\frac{1}{\sqrt{-\operatorname{det} g}} \partial_{\mu}\left(\sqrt{-\operatorname{det} g} g^{\mu v} \partial_{v}\right) .
$$

Here we have used the usual convention in which a subscript,$\mu$ denotes an ordinary partial derivative and ; $\mu$ denotes the covariant derivative.

\section{A.3 Geodesics}

Geodesics are the "shortest possible lines" one can draw in curved geometry. Given a covariant derivative operator $\nabla_{\mu}$, we define a geodesic to be a curve whose tangent vector is parallel propagated along itself, i.e., a curve whose tangent $u^{\mu}$ satisfies the equation

$$
u^{v} u_{; v}^{\mu}=0 .
$$

A massive particle moves along a timelike geodesic, $u_{\mu}$ being its velocity. A massless particle moves along a null geodesic in which case the vector $u_{\mu}$ is null. 


\section{A.4 Isometries and Killing Vectors}

A vector field $k^{\mu}$ that generates one parameter group of isometries, i.e., one parameter group of transformations that leave the metric invariant, is called a Killing vector. A Killing vector $k^{\mu}$ satisfies the Killing equation

$$
k_{(\mu ; v)} \equiv k_{\mu ; v}+k_{v ; \mu}=0 .
$$

It is convenient to represent the Killing vector $k^{\mu}$ as a differential operator $k$,

$$
k=k^{\mu} \partial_{\mu}
$$

For a vector field $k$, local coordinates can be found such that

$$
k=\frac{\partial}{\partial x}
$$

where $x$ is one of these coordinates, e.g., $x^{4} \equiv x$. In such a coordinate system, $k^{\mu}=\delta_{4}^{\mu}$ and

$$
k_{(\mu ; v)}=\frac{\partial g_{\mu v}}{\partial x}=0
$$

Hence, one can say that $k_{\mu}$ is Killing if $g_{\mu \nu}$ is independent of $x$.

\section{Examples}

Consider the spherical coordinate system $(t, r, \theta, \phi)$. Suppose the metric components are independent of $t$ and $\phi$. Then there exist two Killing vectors:

- the generator of time translations $\xi=\partial / \partial t$

$$
\xi^{\mu}=\delta_{0}^{\mu} ; \quad \xi_{\mu}=g_{\mu 0}
$$

- the generator of axial isometries $\psi=\partial / \partial \phi$. In spherical coordinates

$$
\psi^{\mu}=\delta_{\phi}^{\mu} ; \quad \psi_{\mu}=g_{\mu \phi} .
$$

\section{A.5 Constants of Motion}

The following proposition relates Killing vectors to constants of motion.

Proposition 1. Let $\chi^{\mu}$ be a Killing vector field and let $\gamma$ be a geodesic with tangent $u^{\mu}$. Then the quantity $\chi_{\mu} u^{\mu}$ is constant along $\gamma$.

Proof: We have

$$
u^{v}\left(\chi_{\mu} u^{\mu}\right)_{; v}=u^{\mu} u^{v} \chi_{\mu ; v}+\chi_{\mu} u^{v} u_{; v}^{\mu}=0 .
$$

The first term vanishes by the Killing equation (A.13) and the second term vanishes by the geodesic equation (A.12). Hence

$$
u^{v}\left(\chi^{\mu} u_{\mu}\right)_{; v}=0
$$

as was to be proved. 


\section{Examples}

Consider a stationary axially symmetric space, as in the example discussed in appendix A.4 with two Killing vectors, $\xi=\partial / \partial t$ and $\psi=\partial / \partial \phi$, corresponding to time translation and axial isometries, respectively. In addition, assume the asymptotic flatness, i.e.,

$$
g_{\mu \nu} \rightarrow \operatorname{diag}\left(1,-1,-r^{2},-r^{2} \sin ^{2} \theta\right) \quad \text { as } r \rightarrow \infty .
$$

For a particle of mass $m$ moving along a timelike geodesic, the two conserved quantities along the geodesics are

- the energy

$$
E=m u^{\mu} \xi_{\mu}=\xi_{\mu} q^{\mu}=g_{0 \mu} q^{\mu}=\left.q^{0}\right|_{\infty},
$$

- the z-component of the angular momentum

$$
L=-m u^{\mu} \psi_{\mu}=-\psi_{\mu} q^{\mu}=-g_{\phi \mu} q^{\mu}=\left.r^{2} \sin ^{2} \theta q^{\phi}\right|_{\infty} .
$$

\section{A.6 Einstein's Equations}

General relativity relates the geometry of spacetime to matter through Einstein's field equations

$$
R_{\mu v}-\frac{1}{2} g_{\mu v} \mathscr{R}=-8 \pi T_{\mu \nu},
$$

where $T_{\mu \nu}$ is the energy-momentum tensor.

\section{B. Basic Fluid Dynamics}

Consider a perfect gravitating relativistic fluid. We denote by $u_{\mu}, p, \rho, n$, and $\sigma$ the velocity, pressure, energy density, particle number density, and entropy density of the fluid. The energymomentum tensor of a perfect fluid is given by

$$
T_{\mu v}=(p+\rho) u_{\mu} u_{v}-p g_{\mu v}
$$

where $g_{\mu \nu}$ is the metric tensor with the Lorentzian signature $(+---)$. Hence, in this convention, we have

$$
u^{\mu} u_{\mu}=g_{\mu v} u^{\mu} u^{v}=1
$$

The particle number conservation is described by the continuity equation

$$
\left(n u^{\mu}\right)_{; \mu}=\frac{1}{\sqrt{-g}} \partial_{\mu}\left(\sqrt{-g} n u^{\mu}\right)=0 .
$$

The energy-momentum conservation

$$
T_{; v}^{\mu v}=0
$$

applied to (B.1) yields the relativistic generalization of Euler's equation [120]

$$
(p+\rho) u^{v} u_{\mu ; v}-\partial_{\mu} p+u_{\mu} u^{v} \partial_{v} p=0
$$




\section{B.1 Fluid Velocity}

It is convenient to parameterize the four-velocity of the fluid in terms of three-velocity components. To do this, we use the projection operator $g_{\mu v}-t_{\mu} t_{v}$, which projects a vector into the subspace orthogonal to the time-translation Killing vector $\xi^{\mu}=(1 ; \overrightarrow{0})$, where $t_{\mu}$ is the unit vector

$$
t^{\mu}=\frac{\xi^{\mu}}{\sqrt{\xi^{v} \xi_{v}}}=\frac{\delta_{0}^{\mu}}{\sqrt{g_{00}}} ; \quad t_{\mu}=\frac{\xi_{\mu}}{\sqrt{\xi^{v} \xi_{v}}}=\frac{g_{\mu 0}}{\sqrt{g_{00}}} .
$$

We split up the vector $u_{\mu}$ in two parts: one parallel with and the other orthogonal to $t_{\mu}$ :

$$
u_{\mu}=\gamma t_{\mu}+\left(g_{\mu v}-t_{\mu} t_{v}\right) u^{v}
$$

where

$$
\gamma=t^{\mu} u_{\mu}
$$

From (B.7) with (B.6) we find (see, e.g., appendix of [121])

$$
\begin{aligned}
& u^{\mu}=\gamma\left(\frac{1}{\sqrt{g_{00}}}-\frac{g_{0 j} v^{j}}{g_{00}} ; v^{i}\right), \\
& u_{\mu}=\gamma\left(\sqrt{g_{00}} ; \frac{g_{0 i}}{\sqrt{g_{00}}}-v_{i}\right),
\end{aligned}
$$

where

$$
v_{i}=\gamma_{i j} v^{j}, \quad v^{2}=v^{i} v_{i}, \quad \gamma^{2}=\left(1-v^{2}\right)^{-1},
$$

with the induced three-dimensional spatial metric:

$$
\gamma_{i j}=\frac{g_{0 i} g_{0 j}}{g_{00}}-g_{i j} ; \quad i, j=1,2,3 .
$$

Since $u^{\mu}$ and $t^{\mu}$ are timelike unit vectors, a consequence of (B.7) is that $\gamma \geq 1$ and hence $0 \leq v^{2}<1$.

\section{B.2 Hydrostatic Equilibrium}

From Euler's equation (B.5) we can derive the condition of hydrostatic equilibrium. We can use the comoving frame of reference in which the fluid velocity takes the form

$$
u^{\mu}=\frac{\delta_{0}^{\mu}}{\sqrt{g_{00}}} ; \quad u_{\mu}=\frac{g_{\mu 0}}{\sqrt{g_{00}}} .
$$

In equilibrium the metric is static; all components are independent of time, and the mixed components $g_{0 i}$ are zero. Equation (B.5) then gives

$$
(\rho+p) \Gamma_{\mu 0}^{0} u^{0} u_{0}=(\rho+p) \frac{1}{2} g^{00} \partial_{\mu} g_{00}=-\partial_{\mu} p
$$

or

$$
\partial_{\mu} p=-(p+\rho) g_{00}^{-1 / 2} \partial_{\mu} g_{00}^{1 / 2}
$$




\section{Basic Thermodynamics}

Consider a nonrotating fluid consisting of $N$ particles in equilibrium at nonzero temperature. A canonical ensemble is subject to the constraint that the number of particles

$$
\int_{\Sigma} n u^{\mu} d \Sigma_{\mu}=N
$$

should be fixed. The spacelike hypersurface $\Sigma$ that contains the fluid is orthogonal to the timetranslation Killing vector field $\xi^{\mu}$. In equilibrium $\xi^{\mu}$ is related to the velocity of the fluid.

$$
\xi^{\mu}=\xi u^{\mu} ; \quad \xi=\left(\xi^{\mu} \xi_{\mu}\right)^{1 / 2} .
$$

It may be shown that those and only those configurations will be in equilibrium for which the free energy assumes a minimum [122]. The canonical free energy is defined as [122, 123].

$$
F=M-\int_{\Sigma} T \sigma \xi^{\mu} d \Sigma_{\mu}
$$

where $M$ is the total mass as measured from infinity. The entropy density is obtained using the standard thermodynamic relation

$$
\sigma=\frac{1}{T}(p+\rho-\mu n)
$$

\section{C.1 Tolman Equations}

The temperature $T$ and the chemical potential $\mu$ are metric-dependent local quantities. Their spacetime dependence may be derived from the equation of hydrostatic equilibrium (B.14) [10, $120]$ and the thermodynamic identity (Gibbs-Duhem relation)

$$
d \frac{p}{T}=n d \frac{\mu}{T}-\rho d \frac{1}{T}
$$

The crucial condition is that the heat flow and diffusion should vanish [124]

$$
\frac{\mu}{T}=\text { const },
$$

which may be derived from the physical requirement that the rate of entropy change with particle number at fixed energy density should be constant, i.e.,

$$
\left.\frac{\partial \sigma}{\partial n}\right|_{\rho}=\text { const },
$$

where 'const' is independent of $\rho$. From (C.4) and (C.5) we obtain

$$
d \sigma=\frac{1}{T} d \rho-\frac{\mu}{T} d n
$$

and hence

$$
\left.\frac{\partial \sigma}{\partial n}\right|_{\rho}=-\frac{\mu}{T} .
$$


from which (C.6) immediately follows. Next, equation (C.6), together with (B.14) and (C.5), implies the well-known Tolman equations

$$
T g_{00}^{1 / 2}=T_{0} ; \quad \mu g_{00}^{1 / 2}=\mu_{0}
$$

where $T_{0}$ and $\mu_{0}$ are constants equal, respectively, to the temperature and the chemical potential at infinity. In a grand-canonical ensemble, $T_{0}$ and $\mu_{0}$ may be chosen arbitrarily. In a canonical ensemble, $\mu_{0}$ is an implicit functional of $\xi$ because of the constraint (C.1) that the total number of particles should be fixed.

\section{C.2 Fermi Distribution}

Consider a gas consisting of $N$ fermions with the mass $m$ contained within a hypersurface $\Sigma$. The equation of state may be represented in a parametric form using the well-known momentum integrals over the Fermi distribution function [125]

$$
\begin{gathered}
n=g \int_{0}^{\infty} \frac{d^{3} q}{(2 \pi)^{3}} \frac{1}{1+e^{E / T-\mu / T}}, \\
\rho=g \int_{0}^{\infty} \frac{d^{3} q}{(2 \pi)^{3}} \frac{E}{1+e^{E / T-\mu / T}}, \\
p=g T \int_{0}^{\infty} \frac{d^{3} q}{(2 \pi)^{3}} \ln \left(1+e^{-E / T+\mu / T}\right),
\end{gathered}
$$

where $E=\sqrt{m^{2}+q^{2}}$ and $T$ and $\mu$ are the local temperature and the local chemical potential, respectively, defined by Tolman's equations (C.10). By partial integration, the last equation may be written as

$$
p=g \int_{0}^{\infty} \frac{d^{3} q}{(2 \pi)^{3}} \frac{q^{2}}{3 E} \frac{1}{1+e^{E / T-\mu / T}}
$$

The integer $g$ denotes the spin degeneracy factor. In most applications we take $g=2$ (spin up and spin down). Strictly speaking, in each equation (C.11)-(C.14) one should also add the antiparticle term which is of the same form as the corresponding right-hand side of (C.11)-(C.14) with $\mu$ replaced by $-\mu$. However, the contribution of antiparticles in astrophysical objects is almost always negligible ${ }^{8}$.

Equations (C.4) and (C.8) may be combined to yield another useful thermodynamic identity

$$
d w=T d\left(\frac{\sigma}{n}\right)+\frac{1}{n} d p
$$

with $w=(p+\rho) / n$ being the specific enthalpy.

\footnotetext{
${ }^{8}$ One exception is neutrino stars made of Dirac type neutrinos. There, the numbers of neutrinos and antineutrinos are equal and separately conserved, hence $g=4$
} 


\section{C.3 Isentropic Fluid}

Euler's equation is simplified if one restricts consideration to an isentropic flow. A flow is said to be isentropic when the specific entropy $\sigma / n$ is constant, i.e.,when

$$
\partial_{\mu}\left(\frac{\sigma}{n}\right)=0
$$

A flow may in general have a nonvanishing vorticity $\omega_{\mu v}$ defined as

$$
\omega_{\mu v}=h_{\mu}^{\rho} h_{v}^{\sigma} u_{[\rho ; \sigma]},
$$

where

$$
h_{v}^{\mu}=\delta_{v}^{\mu}-u^{\mu} u_{v}
$$

is the projection operator which projects an arbitrary vector in spacetime into its component in the subspace orthogonal to $u^{\mu}$. A flow with vanishing vorticity, i.e., when

$$
\omega_{\mu v}=0,
$$

is said to be irrotational. In the following we assume that the flow is isentropic and irrotational.

As a consequence of equation (C.16) and the thermodynamic identity (C.15), equation (B.5) simplifies to

$$
u^{v}\left(w u_{\mu}\right)_{; v}-\partial_{\mu} w=0
$$

Furthermore, for an isentropic irrotational flow, equation (C.19) implies [126]

$$
\left(w u_{\mu}\right)_{; v}-\left(w u_{v}\right)_{; \mu}=0 .
$$

In this case, we may introduce a scalar function $\varphi$ such that

$$
w u_{\mu}=-\partial_{\mu} \varphi,
$$

where the minus sign is chosen for convenience. Obviously, the quantity $w u^{\mu}$ in the form (C.22) satisfies equation (C.21). Solutions of this form are the relativistic analogue of potential flow in nonrelativistic fluid dynamics [120].

\section{C.4 Degenerate Fermi Gas}

In the limit $T \rightarrow 0$, the Fermi distribution in (C.11)-(C.13) becomes a step function that yields an elementary integral with the upper limit equal to the Fermi momentum

$$
q_{\mathrm{F}}=\sqrt{\mu^{2}-m^{2}}=m X .
$$

The equation of state can be expressed in terms of elementary functions of $X$. With $g=2$, we find

$$
\begin{gathered}
n=2 \int_{0}^{q_{\mathrm{F}}} \frac{d^{3} q}{(2 \pi)^{3}}=\frac{1}{3 \pi^{2}} m^{3} X^{3}, \\
\rho=2 \int_{0}^{q_{\mathrm{F}}} E \frac{d^{3} q}{(2 \pi)^{3}}=\frac{1}{8 \pi^{2}} m^{4}\left[X\left(2 X^{2}+1\right) \sqrt{X^{2}+1}-\operatorname{Arsh} X\right] \\
p=2 \int_{0}^{q_{\mathrm{F}}} \frac{q^{2}}{3 E} \frac{d^{3} q}{(2 \pi)^{3}}=\frac{1}{8 \pi^{2}} m^{4}\left[X\left(\frac{2}{3} X^{2}-1\right) \sqrt{X^{2}+1}+\operatorname{Arsh} X\right] .
\end{gathered}
$$

There are two important limits: 
- Nonrelativistic limit, $X \ll 1$

$$
\begin{aligned}
& \rho=\frac{1}{3 \pi^{2}} m^{4}\left(X^{3}+\frac{3}{10} X^{5}-\frac{3}{56} X^{7} \ldots\right), \\
& p=\frac{1}{15 \pi^{2}} m^{4}\left(X^{5}-\frac{5}{14} X^{7}+\frac{5}{24} X^{9} \ldots\right),
\end{aligned}
$$

Obviously, in this limit, $p \ll \rho$.

- Ultrarelativistic limit

$$
\begin{aligned}
& \rho=\frac{1}{4 \pi^{2}} m^{4}\left(X^{4}+X^{2}-\frac{1}{2} \ln 2 X \ldots\right), \\
& p=\frac{1}{12 \pi^{2}} m^{4}\left(X^{4}-X^{2}+\frac{3}{2} \ln 2 X \ldots\right) .
\end{aligned}
$$

Retaining the dominant terms, these equations yield the well-known equation of state for an ultrarelativistic gas

$$
p=\frac{\rho}{3},
$$

which also holds for massless Bose and Fermi gases at finite temperature.

\section{C.5 Polytropic Gases}

A gas of particles of mass $m$ is called polytropic if the equation of state may be written in the form

$$
p=\mathscr{K} n^{\Gamma},
$$

where $\mathscr{K}$ is a constant that depends on $m$. Using this equation and the thermodynamic identity (C.15) the energy density $\rho$ and the entropy density $\sigma$ may also be expressed in terms of $n$. For an isentropic flow, it follows

$$
\rho=m n+\frac{\mathscr{K}}{\Gamma-1} n^{\Gamma}
$$

A degenerate Fermi gas approaches a polytropic equation of state in both the nonrelativistic and the extreme-relativistic limits:

- Nonrelativistic limit. Retaining only the dominant term in (C.28) we find

$$
p=\frac{3^{2 / 3} \pi^{4 / 3}}{5 m} n^{5 / 3} \text {. }
$$

- Ultrarelativistic limit. In this case, the dominant term in (C.30) yields

$$
p=\frac{3^{1 / 3} \pi^{2 / 3}}{4} n^{4 / 3} .
$$

\section{Acknowledgment}

I thank the organizers of the school for the invitation and hospitality. I am grateful to Andreas Müller for his valuable comments which helped me improve the manuscript. This work was supported by the Ministry of Science and Technology of the Republic of Croatia under Contract No. 0098002. 


\section{References}

[1] R. A. Remillard and J. E. McClintock, X-ray Properties of Black-Hole Binaries, astro-ph/0606352.

[2] L. Ferrarese and H. Ford, Supermassive Black Holes in Galactic Nuclei: Past, Present and Future Research, astro-ph/0411247

[3] C. W. Misner, K. S. Thorne, and J. A. Wheeler, Gravitation, W. H. Freeman and Company, NY 1973.

[4] S.L. Shapiro and S.A. Teukolsky, Black Holes, White Dwarfs and Neutron Stars, Wiley \& Sons, NY 1983

[5] R.M. Wald, General relativity, University of Chicago, Chicago 1984.

[6] B.W. Carroll and D.A. Ostlie, An Introduction to Modern Astrophysics, Addison-Wesley, Reading MA 1996.

[7] P.K. Townsend, Black Holes -Lecture notes, gr-qc/9707012

[8] R. Narayan, Black Holes in Astrophysics, New J. Phys. 7 (2005) 199 [gr-qc/ 0506078 ].

[9] "Physics and Astrophysics of Supermassive Black-Holes", 10 - 14 July 2006, Santa Fe, New Mexico; http://qso.lanl.gov/meetings/meet2006/participate.html

[10] R.C. Tolman, Relativity Thermodynamics and Cosmology, Clarendon, Oxford 1934; Dover, New York 1987.

[11] S. Chandrasekhar, Astrophys. J. 74 (1931) 81.

[12] J.R. Oppenheimer andG.M. Volkoff, On Massive Neutron Cores, Phys. Rev. 55 (1939) 374.

[13] N. Bilic and R.D. Viollier, Gravitational phase transition of fermionic matter in a general-relativistic framework, Eur. Phys. J. C 11 (1999) 173 [hep-ph/9809563].

[14] C.E. Rhodes and R. Ruffini, Phys. Rev. Lett. 32 (1974) 324.

[15] M. Nauenberg and G. Chapline, Astrophys. J. 179 (1973) 277.

[16] J.B. Hartle and A.G. Sabbadini, Astrophys. J. 213 (1977) 831.

[17] J. Hansson, A hierarchy of cosmic compact objects - without black holes, ast ro-ph/0603342.

[18] M. Bojowald et al., A black hole mass threshold from non-singular quantum gravitational collapse, Phys. Rev. Lett. 95 (2005) 091302. [gr-qc/ 0503041 ].

[19] S. Weinberg, Gravitation and Cosmology, Wiley \& Sons, NY 1972.

[20] A. S. Eddington, Nature 113 (1924) 192; D. Finkelstein, Phys. Rev. 110 (1958) 965.

[21] A. J. S. Hamilton and J. P. Lisle, The river model of black holes, gr-qc/0411060.

[22] J.M. Bardeen, W.H. Press and S.A. Teukolski, Astrophys. J. 178 (1972) 347.

[23] R. Penrose, Riv. Nuovo Cim. 1 (1969) 252.

[24] S. W. Hawking, Phis. Rev. Lett. 26 (1971) 1344.

[25] R. Znajek, Nature 262 (1976) 270.

[26] R. D. Blandford and R. Znajek, MNRAS 179 (1977) 433.

[27] S. Koide et al., Science 295 (2002) 1688. 
[28] K. I. Nishikawa et al. A General Relativistic Magnetohydrodynamics Simulation of Jet Formation with a State Transition, Astrophys. J. 625 (2005) 60. [astro-ph/ 0403032 ].

[29] S. Koide, Phys. Rev. D 67 (2003) 104010.

[30] V. Semenov, S. Dyadechkin and B. Punsly, Simulations of jets driven by black hole rotation, Science 305 (2004) 978 [astro-ph/ 0408371 ].

[31] J. P. De Villiers, J. F. Hawley and J. H. Krolik, Magnetically Driven Accretion Flows in the Kerr Metric I: Models and Overall Structure, Astrophys. J. 599 (2003) 1238.

[32] J. C. McKinney and C. F. Gammie, A Measurement of the Electromagnetic Luminosity of a Kerr Black Hole, Astrophys. J. 611 (2004) 977 [astro-ph/ 0404 512].

[33] H. Tananbaum et al., Astrophys. J. Lett. 174 (1972) L143.

[34] B.L. Webster,P. Murdin, Nature 235 (1972) 37.

[35] C.T. Bolton, Nature 235 (1972) 271.

[36] R. P. Fender, Powerful jets from black hole X-ray binaries in Low/Hard X-ray states, MNRAS 322 (2001) 31 [astro-ph/0008447].

[37] B. Paczynski, Astron. Astrophys. 34 (1974) 161.

[38] S. Balbus and J. F. Hawley, The Dynamics of the Magnetoviscous Instability, Astrophys. J. 376 (1991) 214.

[39] T. Islam and S. Balbus, The Dynamics of the Magnetoviscous Instability, Astrophys. J. 633 (2005) 328 [astro-ph/0504666].

[40] A. Müller and M. Camenzind, Relativistic emission lines from accreting black holes - The effect of disk truncation on line profiles, Astron. Astrophys. 413 (2004) 861 [astro-ph / 0309832 ].

[41] R. Genzel et al., Nature 425 (2003) 934.

[42] B. Aschenbach, N. Grosso, D. Porquet and P. Predehl, X-ray flares reveal mass and angular momentum of the Galactic Center black hole, Astron. Astrophys. 417 (2004) 71 [astro-ph/0401589].

[43] Y. Tanaka et al., Nature 375 (1995) 659.

[44] N. I. Shakura and R. A. Sunyaev, Astron. Astrophys. 24 (1973) 337.

[45] C. S. Reynolds ,L. W. Brenneman and D. Garofalo, Black Hole Spin in AGN and GBHCs, in T. Maccarone, R. Fender, and L. Ho (eds.), From X-ray Binaries to Quasars: Black Hole Accretion on All Mass Scales, Astrophys. Space Sci. 300 (2005) 71 [astro-ph/ 0410116 ].

[46] J.M. Miller et al., Astrophys. J. Lett. 606 (2004) L131.

[47] P. J. Armitage and C. S. Reynolds, The variability of accretion onto Schwarzschild black holes from turbulent magnetized discs, MNRAS 341 (2003) 1041 [astro-ph/0302271]; movies at http://jilawww.colorado.edu/ pja/black_hole.html

[48] C. S. Reynolds, talk at Physics and Astrophysics of Supermassive Black-Holes 10 - 14 July 2006, Santa Fe, New Mexico (see ref. [9]).

[49] D. Lynden-Bell, Nature 223 (1969) 690. 
[50] G.M. Madejski, Black holes in active galactic nuclei: observations, in Theory of Black Hole Accretion Discs, eds. M.A. Abramowicz, G. Bjvrnsson and J.E. Pringle, Cambridge University Press, Cambridge 1998 [astro-ph/9903141].

[51] T. de Zeeuw, Evidence for massive black holes in nearby galactic nuclei, astro-ph/ 0009249.

[52] M.J. Rees, Astrophysical evidence for black holes, in R.M. Wald (ed.), Black holes and relativistic stars, Univ. of Chicago Press, Chicago 1996; M.J. Rees, Supermassive black holes: Their formation, and their prospects as probes of relativistic gravity, astro-ph/9912346

[53] F. Melia, Nature 437 (2005) 1105.

[54] D. Merritt and L. Ferrarese, Relationship of black holes to bulges, a stro-ph/0107134.

[55] J. Kormendy and K. Gebhardt, Supermassive black holes in galactic nuclei, ast ro-ph/0105230.

[56] J. Kormendy, The Stellar-Dynamical Search for Supermassive Black Holes in Galactic Nuclei, in L.C. Ho (ed.), Coevolution of Black Holes and Galaxies, Cambridge Univ. Press, Cambridge 2004 [astro-ph/0306353.

[57] F. Macchetto et al., Astrophys. J. 489 (1997) 579.

[58] Z.I. Tsvetanov et al, Morphology of the nuclear disk in M87, in the Proceedings of the M87 Workshop, Ringberg castle, Germany, 15-19 Sep 1997, astro-ph / 9803178.

[59] J. R. Herrnstein et al, VLBA Continuum Observations of NGC 4258: Constraints on an Advection-Dominated Accretion Flow, Astrophys. J. Lett. 497 (1998) L69 [astro-ph/9802264].

[60] J. M. Moran, L. J. Greenhill and J. R. Herrnstein, Observational Evidence for Massive Black Holes in the Centers of Active Galaxies, J. Astrophys. Astron. 20 (1999) 165 [astro-ph/0002085].

[61] W. Pieych and A. M. Read, Astron. Astrophys. 384 (2002) 793.

[62] A. E. Bragg et al., Accelerations of Water Masers in NGC4258, Astrophys. J. 535 (2000) 73 [astro-ph/0001543].

[63] R. Schödel et al., Nature 419 (2002) 694.

[64] F. Eisenhauer et al., Astrophys. J. Lett. 597 (2003) L121.

[65] A. Eckart et al., MNRAS 331 (2002) 917.

[66] A. Ghez et al.: Astrophys. J. 509 (1998) 678.

[67] A. Ghez et al., invited talk at the Galactic Center conference, 4 - 8 October 2002, Kalua-Kona (Hawaii); A. Ghez et al, Astrophys. J. Lett. 586 (2003) L127.

[68] A. Ghez, movie at http: //www. astro.ucla.edu/ ghezgroup/gc/pictures/

[69] L. Ferrarese and D. Merritt, Supermassive black holes, Phys. World 15N6 (2002) 41 [astro-ph/0206222].

[70] M. Elvis, G. Risaliti, and G. Zamorani, Astrophys. J. Lett. 565 (2002) L75.

[71] Q. Yu and S. Tremaine, MNRAS 335 (2002) 965.

[72] R. Mushotzky, Ultra-Luminous Sources in Nearby Galaxies, Prog. Theor. Phys. Suppl. 155 (2004) 27 [astro-ph/0411040].

[73] E. J. M. Colbert and R. F. Mushotzky, The Nature of Accreting Black Holes in Nearby Galaxy Nuclei, Astrophys. J. 519 (1999) 89 [astro-ph/9901023]. 
[74] J. Poutanen, S. Fabrika, A. G. Butkevich and P. Abolmasov, On the nature of ultraluminous X-ray sources, or what a black hole should look like, ast ro-ph/0609274.

[75] M. A. Abramowicz, W. Kluzniak and J. P. Lasota, No observational proof of the black-hole event-horizon, Astron. Astrophys. 396 (2002) L31 [astro-ph/ 2207270 ].

[76] S. A. Hughes, Trust but verify: The case for astrophysical black holes, eConf C0507252 (2005) L006 [hep-ph/0511217].

[77] R. Narayan and I. s. Yi, Advection dominated accretion: A Selfsimilar solution, Astrophys. J. 428 (1994) L13 [astro-ph/9403052].

[78] R. Narayan, I. Yi and R. Mahadevan, Advection Dominated Accretion Model For Sagittarius A: Evidence For A 10**6 Solar Mass Black Hole At The Galactic Center, Nature 374 (1995) 623 [astro-ph/9411060].

[79] R. Narayan, M.R. Garcia, and J.E. McClintock, Astrophys. J. Lett. 478 (1997) L79.

[80] R. Narayan, J.S. Heyl, Astrophys. J. Lett. 574 (2002) L139.

[81] R. Narayan and J. S. Heyl, Astrophys. J. 599 (2003) 419.

[82] H. Falcke, F. Melia, and E. Agol, Astrophys. J. 528 (2000) L13.

[83] T. P. Krichbaum et al., Sub-milliarcsecond Imaging of SgrA* and M87, a st ro-ph/ 0607072.

[84] E. Maoz, Dynamical Constraints On Alternatives To Massive Black Holes In Galactic Nuclei, Astrophys. J. Lett. 494 (1998) L181 [astro-ph/9710309].

[85] R.D. Viollier, D. Trautmann and G.B. Tupper, Phys. Lett. B306 (1993) 79.

[86] R.D. Viollier, Prog. Part. Nucl. Phys. 32 (1994) 51.

[87] N. Bilić, F. Munyaneza and R.D. Viollier, Phys. Rev. D59 (1999) 024003.

[88] N. Bilić, R.L. Lindebaum, G.B. Tupper and R.D. Viollier, Phys. Lett. B515 (2001) 105.

[89] F.Munyaneza, D. Tsiklauri and R.D. Viollier, Astrophys. J. 509 (1998) L105; ibid. 526 (1999) 744.

[90] K. Abazajian and S. M. Koushiappas, Constraints on sterile neutrino dark matter, Phys. Rev. D 74 (2006) 023527 [astro-ph/0605271].

[91] A. Kusenko, Detecting sterile dark matter in space, astro-ph/0608096.

[92] N. Bilic, G.B. Tupper, and R.D. Viollier, Sterile Neutrino Dark Matter in the Galaxy, in J. Trampetic and J. Wess (eds.), Particle Physics and the Universe, Proceedings of the 9th Adriatic Meeting, Dubrovnik, 2003, Springer Proceedings in Physics 98, Berlin 2004 [astro-ph / 0310294 ].

[93] N. Bilić and H. Nikolić, Self-gravitating bosons at nonzero temperature, Nucl. Phys. B 590 (2000) 575 [gr-qc/0006065].

[94] D.J. Kaup, Phys. Rev. 172 (1968) 1331.

[95] R. Friedberg, T.D. Lee and Y. Pang, Phys. Rev. D 35 (1987) 3640.

[96] P. Jetzer, Phys. Rep. 220 (1992) 163.

[97] F.E. Schunck and E.W. Mielke, Class. Quant. Grav. 20 (2003) R301.

[98] E. Seidel and W.-M. Suen, Phys. Rev. Lett. 72 (1994) 2516.

[99] E.W. Mielke and F.E. Schunck, Nucl. Phys. B 564 (2000) 185. 
[100] M. Colpi, S. L. Shapiro and I. Wasserman, Boson stars: Gravitational equilibria of self-interacting scalar fields, Phys. Rev. Lett. 57 (1986) 2485.

[101] E. W. Mielke and F. E. Schunck, Boson stars: Early history and recent prospects, gr-qc/9801063.

[102] R. R. Caldwell, A Phantom Menace?, Phys. Lett. B 545 (2002) 23 [astro-ph/9908168].

[103] R. R. Caldwell, M. Kamionkowski and N. N. Weinberg, Phantom Energy and Cosmic Doomsday Phys. Rev. Lett. 91 (2003) 071301 [astro-ph/0302506].

[104] G. Chapline, Dark energy stars, eConf C041213 (2004) 0205 [astro-ph/0 503200 ].

[105] F. S. N. Lobo, Stable dark energy stars, Class. Quant. Grav. 23 (2006) 1525 [gr-qc/050 8115 ].

[106] N. Bilić, G.B. Tupper and R.D. Viollier, Phys. Lett. B 535 (2002) 17.

[107] C. Chapline et al., Phil. Mag. B 81 (2001) 235.

[108] C. Chapline et al., Int. J. Mod. Phys. A 18 (2003) 3587.

[109] P.O. Mazur and E. Mottola, Gravitational Condensate Stars: An Alternative to Black Holes, gr-qc/0109035.

[110] P. O. Mazur and E. Mottola, Gravitational vacuum condensate stars, Proc. Nat. Acad. Sci. 1119545 (2004) [gr-qc/0407075].

[111] I. Dymnikova, Spherically symmetric spacetime with the regular de Sitter center, Int. J. Mod. Phys. D 12, 1015 (2003) [gr-qc/ 0304110$].$

[112] M. Visser and D.L. Wiltshire, Class. Quant. Grav. 21 (2004) 1135.

[113] B. M. N. Carter, Stable gravastars with generalised exteriors, Class. Quant. Grav. 22 (2005) 4551 [gr-qc/0509087].

[114] W. Israel, Nuovo Cim. B 44 S10 (1966) 1; Erratum-ibid B 48 (1967) 463.

[115] A. Kamenshchik, U. Moschella and V. Pasquier, Phys. Lett. B 511 (2001) 265.

[116] N. Bilić, G. B. Tupper and R. D. Viollier, Born-Infeld phantom gravastars, JCAP 0602 (2006) 013 [astro-ph/0503427].

[117] C. Cattoen, T. Faber and M. Visser, Gravastars must have anisotropic pressures, Class. Quant. Grav. 22 (2005) 4189 [gr-qc/ 0505137 ].

[118] A. DeBenedictis et al, Gravastar solutions with continuous pressures and equation of state, Class. Quant. Grav. 23 (2006) 2303. [gr-qc/0511097].

[119] L.D. Landau, E.M. Lifshitz, The classical theory of fields, Pergamon, Oxford 1994.

[120] L.D. Landau, E.M. Lifshitz, Fluid Mechanics, Pergamon, Oxford, 1993.

[121] N. Bilić, Relativistic Acoustic Geometry, Class. Quant. Grav. 16 (1999) 3953. [gr-qc/9908002].

[122] N. Bilić and R. D. Viollier, General-relativistic Thomas-Fermi model, Gen. Rel. Grav. 31 (1999) 1105 [gr-qc/9903034].

[123] G.W. Gibbons and S.W. Hawking, Action Integrals and Partition Function in Quantum Gravity, Phys. Rev. D 55 (1977) 2752.

[124] W. Israel, Ann. Phys. 100 (1976) 310.

[125] J. Ehlers, Survey of General Relativity Theory, in W. Israel (ed.), Relativity, Astrophysics and Cosmology, D. Reidel Publishing Company, Dordrecht/Boston 1973.

[126] A.H. Taub, Relativistic Fluid Mechanics, Ann. Rev. Fluid Mech. 10 (1978) 301. 